\author{
K. Topolnicki • J. Golak • R. Skibiński • A. E. Elmeshneb \\ W. Glöckle • A. Nogga • H. Kamada
}

\title{
Deuteron Disintegration in Three Dimensions
}

Received: 15 June 2012 / Accepted: 23 July 2012 / Published online: 22 August 2012

(C) The Author(s) 2012. This article is published with open access at Springerlink.com

\begin{abstract}
We compare results from traditional partial wave treatment of deuteron electro-disintegration with a new approach that uses three-dimensional formalism. The new framework for the two-nucleon ( $2 \mathrm{~N})$ system using a complete set of isospin-spin states made it possible to construct simple implementations that employ a very general operator form of the current operator and $2 \mathrm{~N}$ states.
\end{abstract}

\section{Introduction}

The theoretical description of electromagnetic processes is based, like most nuclear physics formalisms, on a partial wave decomposition of relevant operators. This restricts theoretical methods to systems, where a relatively small number of partial waves are important. Recently formalisms of three-dimensional (3D) description of few-body have systems and processes have been extensively developed. In this paper we fill a gap in the development of 3D framework and present an approach that allows for a simple implementation of 3D electromagnetic currents. Our final expressions can be translated to a numerical implementation via direct substitutions of 16-dimensional square matrices representing operators in the $2 \mathrm{~N}$ isospin-spin space. Finding the matrix representation of relevant operators is greatly simplified by using symbolic programming in the Mathematica ${ }^{\circledR}$ [1] software package. Our approach allows us to use a very general operator form of current operators and can therefore be used for a wide class of processes.

In this paper we apply it to the case of electron induced deuteron disintegration and compare new results with traditional partial wave calculations. It is worth noting that the methods presented in the following sections can be applied to the description of other processes, where electroweak probes interact with the $2 \mathrm{~N}$ system. Electromagnetic currents can be replaced by any operators acting on the same degrees of freedom; this makes

Dedicated to Prof. H. Witała on the occasion of his 60th birthday.

K. Topolnicki $(\varangle) \cdot$ J. Golak · R. Skibiński · A. E. Elmeshneb

M. Smoluchowski Institute of Physics, Jagiellonian University, 30059 Kraków, Poland

E-mail: kacper.topolnicki@uj.edu.pl

W. Glöckle

Institut für Theoretische Physik II, Ruhr-Universität Bochum, 44780 Bochum, Germany

\footnotetext{
A. Nogga for Hadron Physics, 52425 Jülich, Germany

H. Kamada

Department of Physics, Faculty of Engineering, Kyushu Institute of Technology,

1-1 Sensuicho Tobata, Kitakyushu 804-8550, Japan
}

Forschungszentrum Jülich, Institut für Kernphysik, Institute for Advanced Simulation and Jülich Center 
our implementations also useful for calculations involving for example muon capture or neutrino induced deuteron disintegration, performed recently still with the use partial wave expansion [2-4].

\section{Formalism and Notation}

We adopt a notation in which capital letters describe the total momentum of a two particle system $\left(\mathbf{P}=\mathbf{p}_{1}+\mathbf{p}_{2}\right)$, lower-case letters describe the relative momentum $\left(\mathbf{p}=\frac{1}{2}\left(\mathbf{p}_{1}-\mathbf{p}_{2}\right)\right)$. Lower indices denote individual particles and upper indices assign a momentum to a particular quantum eigenstate. The two particle momentum eigenstates are normalized such that:

$$
\begin{array}{r}
\left\langle\mathbf{p}^{\prime} \mathbf{P}^{\prime} \mid \mathbf{p} \mathbf{P}\right\rangle=\delta^{3}\left(\mathbf{p}^{\prime}-\mathbf{p}\right) \delta^{3}\left(\mathbf{P}^{\prime}-\mathbf{P}\right), \\
\int d^{3} \mathbf{p} d^{3} \mathbf{P}|\mathbf{p} \mathbf{P}\rangle\langle\mathbf{p} \mathbf{P}|=\mathbf{1}
\end{array}
$$

and the transition from the total and relative to the individual momenta can be achieved using:

$$
\begin{aligned}
& \mathbf{p}_{1}=\mathbf{p}+\frac{1}{2} \mathbf{P}, \\
& \mathbf{p}_{2}=\frac{1}{2} \mathbf{P}-\mathbf{p},
\end{aligned}
$$

where in (3) and in the following we assume that the difference between the proton and neutron mass is negligible.

We examine the case of deuteron disintegration $\left(e+{ }^{2} \mathrm{H} \rightarrow e+p+n\right)$ where the $2 \mathrm{~N}$ system is treated in the non-relativistic approximation. In the initial state the deuteron is at rest $(\mathbf{P}=0)$ and the electron has a momentum magnitude of $q_{e}$. We assume that the rest mass of the electron is negligible in comparison to its kinetic energy; therefore the initial electron energy $E_{e} \approx q_{e}$. The final electron momentum magnitude is $q_{e}^{\prime}$, the final energy $E_{e}^{\prime} \approx q_{e}^{\prime}$ and the electron scattering angle is $\theta_{e}$. The magnitude of the three momentum transferred to the $2 \mathrm{~N}$ system is

$$
Q=\sqrt{q_{e}^{2}+q_{e}^{\prime 2}-2 q_{e} q_{e}^{\prime} \cos \theta_{e}} \approx \sqrt{E_{e}^{2}+E_{e}^{\prime 2}-2 E_{e} E_{e}^{\prime} \cos \theta_{e}}
$$

We work in a reference frame, where the momentum transfer is parallel to $\hat{\mathbf{z}}$. In this frame momentum conservation leads to the expression for the total momentum of the proton and the neutron in the final state:

$$
\mathbf{P}^{\mathrm{f}}=\mathbf{p}_{1}+\mathbf{p}_{2}=(0,0, Q)
$$

The magnitude of the final relative momentum can be calculated from the energy conservation:

$$
p^{\mathrm{f}} \equiv\left|\mathbf{p}^{\mathrm{f}}\right|=\left|\frac{1}{2}\left(\mathbf{p}_{1}-\mathbf{p}_{2}\right)\right|=\frac{1}{2} \sqrt{4 m\left(E_{d}+E_{e}-E_{e}^{\prime}\right)-Q^{2}}
$$

where $E_{d}$ is the (negative) deuteron binding energy, $m$ is the nucleon mass and the direction of $\mathbf{p}^{\mathrm{f}}$ can be arbitrary. The crucial nuclear matrix element $\mathbf{M}^{\mu}$ between the initial deuteron state (where the total momentum $\mathbf{P}=0$ and the two particle total angular momentum has a $\hat{z}$ projection $m_{d}$ ) and the final $2 \mathrm{~N}$ scattering state can be expressed in terms of the full $2 \mathrm{~N}$ current operator $\left(j_{2 N}^{\mu}\right)$ and the $t$ operator:

$$
\begin{aligned}
\mathbf{M}^{\mu}\left(\mathbf{p}^{\mathrm{f}}, \mathbf{P}^{\mathrm{f}}\right) \equiv & { }_{a}\left\langle\mathbf{p}^{\mathrm{f}} \mathbf{P}^{\mathrm{f}}, m_{1} v_{1}, m_{2} v_{2}\left|\left(\mathbf{1}+t(E) G_{0}(E)\right) j_{2 N}^{\mu}\right| \phi_{d} m_{d} \mathbf{P}=0\right\rangle \\
= & { }_{a}\left\langle\mathbf{p}^{\mathrm{f}} \mathbf{P}^{\mathrm{f}}, m_{1} v_{1}, m_{2} v_{2}\right|\left(\mathbf{1}+t(E) G_{0}(E)\right) \\
& \times\left(j^{\mu}(1)+j^{\mu}(2)+j^{\mu}(1,2)\right)\left|\phi_{d} m_{d} \mathbf{P}=0\right\rangle \\
= & 2{ }_{a}\left\langle\mathbf{p}^{\mathrm{f}} \mathbf{P}^{\mathrm{f}}, m_{1} v_{1}, m_{2} v_{2}\left|j^{\mu}(2)\right| \phi_{d} m_{d} \mathbf{P}=0\right\rangle \\
& +{ }_{a}\left\langle\mathbf{p}^{\mathrm{f}} \mathbf{P}^{\mathrm{f}}, m_{1} v_{1}, m_{2} v_{2}\left|j^{\mu}(1,2)\right| \phi_{d} m_{d} \mathbf{P}=0\right\rangle \\
& +{ }_{a}\left\langle\mathbf{p}^{\mathrm{f}} \mathbf{P}^{\mathrm{f}}, m_{1} v_{1}, m_{2} v_{2}\left|t(E) G_{0}(E) j_{2 N}^{\mu}\right| \phi_{d} m_{d} \mathbf{P}=0\right\rangle . \\
&
\end{aligned}
$$


In this equation the final state is anti-symmetrized:

$$
{ }_{a}\left\langle\mathbf{p P}, m_{1} v_{1}, m_{2} v_{2}\right| \equiv \frac{1}{2}\left(\left\langle\mathbf{p P}, m_{1} v_{1}, m_{2} v_{2}\right|-\left\langle-\mathbf{p P}, m_{2} v_{2}, m_{1} v_{1}\right|\right),
$$

where in the first term on the right hand side of (8) particle 1 has a spin (isospin) $\hat{\mathbf{z}}$ projection $m_{1}\left(v_{1}\right)$, particle 2 has a spin (isospin) $\hat{\mathbf{z}}$ projection $m_{2}\left(v_{2}\right)$ and in the second term on the right hand side of (8) the particles are exchanged. The $j(1)(j(2))$ operator is a single nucleon current acting on the degrees of freedom of the first (second) nucleon. The $j(1,2)$ operator accounts for processes where two nucleons are involved, $t$ is the $2 \mathrm{~N}$ transition operator and $G_{0}$ is the free $2 \mathrm{~N}$ propagator. The energy argument of the transition operator and the propagator is $E=\frac{\left(\mathbf{p}^{\mathrm{f}}\right)^{2}}{m}$. Finally, the $\mu$ index denotes the component of the current operator. In particular $\mu=0$ stands for charge density operators, while $\mu=1,2,3$ stand for spatial components. In the following sections the way we calculate the individual parts of $\mathbf{M}^{\mu}$ in Eq. (7) will be discussed separately for a specific choice of the coordinate system and value of $\mu$; we will drop $\mu$ for brevity.

\section{Deuteron Bound State}

The structure of the deuteron wave function can be written in the operator form, following [5-7]:

$$
\begin{aligned}
\left|\phi_{d} m_{d} \mathbf{P}=0\right\rangle= & \int d^{3} \mathbf{p}|\mathbf{p} \mathbf{P}=0\rangle \\
& \times \sum_{l=1}^{2} \phi_{l}(|\mathbf{p}|)\left[\mathbf{1}^{\text {isospin }} \otimes b_{l}(\mathbf{p})^{\mathrm{spin}}\right]\left[|00\rangle \otimes \chi\left(m_{d}\right)\right] \\
\equiv & \int d^{3} \mathbf{p}|\mathbf{p} \mathbf{P}=0\rangle \sum_{l=1}^{2} \phi_{l}(|\mathbf{p}|)\left[B_{l}(\mathbf{p})\right]\left[|00\rangle \otimes \chi\left(m_{d}\right)\right],
\end{aligned}
$$

where

$$
\begin{aligned}
& b_{1}=\mathbf{1}, \\
& b_{2}=\left(\boldsymbol{\sigma}(1) \cdot \mathbf{p} \boldsymbol{\sigma}(2) \cdot \mathbf{p}-\frac{1}{3} \mathbf{p} \cdot \mathbf{p} \mathbf{1}\right) .
\end{aligned}
$$

In Eq. (9) $\left|\chi\left(m_{d}\right)\right\rangle$ is a state in which the spins of the two spin $\frac{1}{2}$ particles are coupled to a total spin 1 with a $\hat{\mathbf{z}}$ projection $m_{d}$. The isospins of the two nucleons are coupled to the total isospin 0 state $|00\rangle . \phi_{l}$ are scalar functions of the relative momentum and $\sigma(1), \sigma(2)$ are doubled spin operators in the spin space of one nucleon and identity operators in the spin space of the other nucleon, respectively:

$$
\begin{aligned}
& \boldsymbol{\sigma}(1)=\left(\sigma^{x} \otimes \mathbf{1}, \sigma^{y} \otimes \mathbf{1}, \sigma^{z} \otimes \mathbf{1}\right), \\
& \boldsymbol{\sigma}(2)=\left(\mathbf{1} \otimes \sigma^{x}, \mathbf{1} \otimes \sigma^{y}, \mathbf{1} \otimes \sigma^{z}\right) .
\end{aligned}
$$

Vector components in (10) and (11) are operators written in terms of the tensor product $\otimes$. They act in the 4-dimensional spin space of the two nucleon system and can be represented by $4 \times 4$ matrices - tensor products of identity operators and Pauli matrices.

We make one step further and combine the isospin-spin states and isospin-spin operators (written inside square brackets [...]), implementing them in terms of 16-dimensional vectors and $16 \times 16$ matrices. In Sect. 7 we will give explicit examples of our numerical treatment.

Scalar functions $\phi_{l}$ in expansion (9) can be calculated using 3D formalism, see for example [7]. Nowadays deuteron bound state calculations can use any $2 \mathrm{~N}$ potentials given in the operator form and do not require substantial computational resources. 


\section{Single Nucleon Currents in Three Dimensions}

Single nucleon $(1 \mathrm{~N})$ currents act on the degrees of freedom of one particle. Their matrix elements in the momentum space depend only on the initial and final momenta and are operators in the isospin-spin space. For example the matrix element for the second nucleon, $j(2)$, reads:

$$
\left[\left\langle\mathbf{p}_{1}^{\prime} \mathbf{p}_{2}^{\prime}|j(2)| \mathbf{p}_{1} \mathbf{p}_{2}\right\rangle\right]=\delta\left(\mathbf{p}_{1}^{\prime}-\mathbf{p}_{1}\right)\left[j\left(2, \mathbf{p}_{2}^{\prime}-\mathbf{p}_{2}, \mathbf{p}_{2}^{\prime}+\mathbf{p}_{2}\right)\right]
$$

where in view of the standard nonrelativistic current, the dependence on the difference and sum of the initial and final momenta is used. Implementing the transition from the individual particle momenta to the relative momenta leads to

$$
\begin{aligned}
& {\left[\left\langle\mathbf{p}^{\prime} \mathbf{P}^{\prime}|j(2)| \mathbf{p P}\right\rangle\right]=\delta\left(\frac{1}{2} \mathbf{P}^{\prime}-\frac{1}{2} \mathbf{P}+\mathbf{p}^{\prime}-\mathbf{p}\right)} \\
& \quad \times\left[j\left(2, \frac{1}{2} \mathbf{P}^{\prime}-\frac{1}{2} \mathbf{P}-\mathbf{p}^{\prime}+\mathbf{p}, \frac{1}{2} \mathbf{P}^{\prime}+\frac{1}{2} \mathbf{P}-\mathbf{p}^{\prime}-\mathbf{p}\right)\right] .
\end{aligned}
$$

Again, the expressions inside the square brackets [... ] can be easily represented using the notion of the Kronecker product, as it will be seen in Sect. 7. The action of $j(2)$ on the deuteron state at rest can be worked out:

$$
\begin{aligned}
& {\left[\left\langle\mathbf{p}^{\prime} \mathbf{P}^{\prime}|j(2)| \phi_{d} m_{d} \mathbf{P}=0\right\rangle\right]=\sum_{l=1}^{2} \phi_{l}\left(\left|\mathbf{p}^{\prime}+\frac{1}{2} \mathbf{P}^{\prime}\right|\right)\left[j\left(2, \mathbf{P}^{\prime},-2 \mathbf{p}^{\prime}\right)\right]} \\
& \quad \times\left[B_{l}\left(\mathbf{p}^{\prime}+\frac{1}{2} \mathbf{P}^{\prime}\right)\right]\left[|00\rangle \otimes \chi\left(m_{d}\right)\right] \\
& \equiv\left[O^{1 \mathrm{~N}}\left(2, \mathbf{p}^{\prime}, \mathbf{P}^{\prime}\right)\right]\left[|00\rangle \otimes \chi\left(m_{d}\right)\right],
\end{aligned}
$$

where we used $\mathbf{P}=0$ and the normalization of momentum eigenstates (1), (2) so $O^{1 \mathrm{~N}}$ is the resulting single particle operator. Equation (14) gives the full isospin-spin state for the final $\mathbf{p}^{\prime}, \mathbf{P}^{\prime}$ momenta.

\section{2N Currents in Three Dimensions}

For a wide class of $2 \mathrm{~N}$ current operators, their matrix elements in the momentum space (operators in isospinspin space) are given in the form:

$$
\left[\left\langle\mathbf{p}_{1}^{\prime} \mathbf{p}_{2}^{\prime}|j(1,2)| \mathbf{p}_{1} \mathbf{p}_{2}\right\rangle\right]=\left[j\left(1,2, \mathbf{p}_{1}^{\prime}-\mathbf{p}_{1}, \mathbf{p}_{2}^{\prime}-\mathbf{p}_{2}\right)\right],
$$

see for example [8-10]. The right hand side of (15) is written as a linear combination of scalar functions and products of spin space operators $(O)$ and isospin space operators $(T)$ :

$$
\begin{gathered}
{\left[j^{0}(1,2)\right]=\sum_{i=1}^{5} \sum_{j=1}^{8} f_{i}^{j S}\left(\mathbf{p}_{1}^{\prime}-\mathbf{p}_{1}, \mathbf{p}_{2}^{\prime}-\mathbf{p}_{2}\right)\left[T_{i} O_{j S}\right],} \\
{[\mathbf{j}(1,2)]=\sum_{i=1}^{5} \sum_{j=1}^{24} f_{i}^{j}\left(\mathbf{p}_{1}^{\prime}-\mathbf{p}_{1}, \mathbf{p}_{2}^{\prime}-\mathbf{p}_{2}\right)\left[T_{i} \mathbf{O}_{j}\right] .}
\end{gathered}
$$

In Ref. [11] even a general operator basis for the local $2 \mathrm{~N}$ current operator was introduced. In Sect. 7 we will show some examples of our dealing with isospin-spin operators (written inside square brackets [...] in Eqs. (16) and (17)).

Again, using (3), current matrix elements become:

$$
\left[\left\langle\mathbf{p}^{\prime} \mathbf{P}^{\prime}|j(1,2)| \mathbf{p} \mathbf{P}\right\rangle\right]=\left[j\left(1,2, \frac{1}{2} \mathbf{P}^{\prime}-\frac{1}{2} \mathbf{P}+\mathbf{p}^{\prime}-\mathbf{p}, \frac{1}{2} \mathbf{P}^{\prime}-\frac{1}{2} \mathbf{P}-\mathbf{p}^{\prime}+\mathbf{p}\right)\right] .
$$

In the following we restrict ourselves to this class of momentum dependences. Our approach can, however, be generalized to include any type of momentum dependence. The action of $j(1,2)$ on the deuteron state can 
be worked out using (1), (2), (3) and the assumption about the total momentum of the initial deuteron state $(\mathbf{P}=0)$. In the laboratory frame it yields:

$$
\begin{aligned}
& {\left[\left\langle\mathbf{p}^{\prime} \mathbf{P}^{\prime}|j(1,2)| \phi_{d} m_{d} \mathbf{P}=0\right\rangle\right]} \\
& =\int d^{3} \mathbf{p}^{\prime \prime} \sum_{l=1}^{2} \phi_{l}\left(\left|\mathbf{p}^{\prime \prime}\right|\right)\left[j\left(1,2, \frac{1}{2} \mathbf{P}^{\prime}+\mathbf{p}^{\prime}-\mathbf{p}^{\prime \prime}, \frac{1}{2} \mathbf{P}^{\prime}-\mathbf{p}^{\prime}+\mathbf{p}^{\prime \prime}\right)\right] \\
& \quad \times\left[B_{l}\left(\mathbf{p}^{\prime \prime}\right)\right]\left[|00\rangle \otimes \chi\left(m_{d}\right)\right] \\
& \equiv\left[O^{2 \mathrm{~N}}\left(1,2, \mathbf{p}^{\prime}, \mathbf{P}^{\prime}\right)\right]\left[|00\rangle \otimes \chi\left(m_{d}\right)\right]
\end{aligned}
$$

where $O^{2 \mathrm{~N}}$ is the resulting two-particle operator. Equation (19) gives the full isospin-spin state for the final $\mathbf{p}^{\prime}, \mathbf{P}^{\prime}$ momenta.

\section{$6 t$ Operator in Three Dimensions}

The $t$ operator satisfies the Lippmann-Schwinger equation:

$$
t(E)=V+t(E) G_{0}(E) V
$$

or

$$
t(E)=V+V G_{0}(E) t(E)
$$

where $G_{0}(E)$ is the free propagator depending on the energy $E$ and $V$ is a $2 \mathrm{~N}$ potential. It follows that, as shown in [12], also $t$ can be written as a linear combination of scalar functions $\left(t_{\gamma, i}\right)$ and operators $\left(\mathbf{W}_{\gamma, i}\right)$ in the isospin-spin space:

$$
\left[\left\langle\mathbf{p}^{\prime}|t(E)| \mathbf{p}\right\rangle\right]=\sum_{\gamma} \sum_{i=1}^{6} t_{\gamma, i}\left(\left|\mathbf{p}^{\prime}\right|,|\mathbf{p}|, \hat{\mathbf{p}}^{\prime} \cdot \hat{\mathbf{p}}, E\right)\left[\mathbf{W}_{\gamma, i}\left(\mathbf{p}^{\prime}, \mathbf{p}\right)\right] .
$$

Here

$$
\left[\mathbf{W}_{\gamma, i}\left(\mathbf{p}^{\prime}, \mathbf{p}\right)\right]=\left[\mathbf{C}_{\gamma}^{\mathrm{isospin}} \otimes \mathbf{w}_{i}^{\text {spin }}\left(\mathbf{p}^{\prime}, \mathbf{p}\right)\right]
$$

are again operators in the isospin-spin space (matrix elements between momentum states), with $\mathbf{w}_{i}(i=$ $1,2, \ldots, 6)$ acting in the 4-dimensional spin space of the $2 \mathrm{~N}$ system. Decomposition (22) is not unique; our choice of the six $\mathbf{w}_{i}$ operators is consistent with [7]:

$$
\begin{array}{r}
\mathbf{w}_{1}\left(\mathbf{p}^{\prime}, \mathbf{p}\right)=\mathbf{1}, \\
\mathbf{w}_{2}\left(\mathbf{p}^{\prime}, \mathbf{p}\right)=\sigma(1) \cdot \sigma(2), \\
\mathbf{w}_{3}\left(\mathbf{p}^{\prime}, \mathbf{p}\right)=i(\boldsymbol{\sigma}(1)+\sigma(2)) \cdot\left(\mathbf{p} \times \mathbf{p}^{\prime}\right), \\
\mathbf{w}_{4}\left(\mathbf{p}^{\prime}, \mathbf{p}\right)=\sigma(1) \cdot\left(\mathbf{p} \times \mathbf{p}^{\prime}\right) \boldsymbol{\sigma}(2) \cdot\left(\mathbf{p} \times \mathbf{p}^{\prime}\right), \\
\mathbf{w}_{5}\left(\mathbf{p}^{\prime}, \mathbf{p}\right)=\sigma(1) \cdot\left(\mathbf{p}^{\prime}+\mathbf{p}\right) \boldsymbol{\sigma}(2) \cdot\left(\mathbf{p}^{\prime}+\mathbf{p}\right), \\
\mathbf{w}_{6}\left(\mathbf{p}^{\prime}, \mathbf{p}\right)=\sigma(1) \cdot\left(\mathbf{p}^{\prime}-\mathbf{p}\right) \boldsymbol{\sigma}(2) \cdot\left(\mathbf{p}^{\prime}-\mathbf{p}\right) .
\end{array}
$$

Scalar functions arising in the decomposition of $t$ can be calculated in the 3D formalism. Calculations can be performed for any type of the NN potential satisfying a similar expansion (22). For details see Ref. [7]. The $C_{\gamma}=|\gamma\rangle\langle\gamma|$ isospin operators project onto one of the four $2 \mathrm{~N}$ isospin states:

$$
|\gamma\rangle=\left|\left(\frac{1}{2} \frac{1}{2}\right) t=0,1 m_{t}=-t \ldots t\right\rangle
$$

where the $|\gamma\rangle$ states are chosen in this way, because $t$ conserves the total $2 \mathrm{~N}$ isospin. 
The rescattering part of the matrix element $\mathbf{M}$ in (7) can be written as:

$$
\begin{aligned}
& {\left[\left\langle\mathbf{p}^{\prime} \mathbf{P}^{\prime}\left|t(E) G_{0}(E) \mathbf{j}_{2 N}\right| \phi_{d} m_{d} \mathbf{P}=0\right\rangle\right]} \\
& =\int d^{3} \mathbf{p}\left[\left\langle\mathbf{p}^{\prime}|t(E)| \mathbf{p}\right\rangle\right] \frac{1}{E-\frac{\mathbf{p}^{2}}{m}+i \epsilon} \\
& \quad\left[O\left(\mathbf{p}, \mathbf{P}^{\prime}\right)\right]\left[|00\rangle \otimes \chi\left(m_{d}\right)\right] \\
& =m \int_{0}^{\bar{p}} \frac{\mathbf{p}^{2}[\mathbf{f}(|\mathbf{p}|)]-\mathbf{p}^{\prime 2}\left[\mathbf{f}\left(\left|\mathbf{p}^{\prime}\right|\right)\right]}{\mathbf{p}^{\prime 2}-\mathbf{p}} d|\mathbf{p}| \\
& \quad+m \frac{\mathbf{p}^{\prime}\left[\mathbf{f}\left(\left|\mathbf{p}^{\prime}\right|\right)\right]}{2}\left(\ln \left(\frac{\bar{p}+\left|\mathbf{p}^{\prime}\right|}{\bar{p}-\left|\mathbf{p}^{\prime}\right|}\right)-i \pi\right) \\
& \quad\left[|00\rangle \otimes \chi\left(m_{d}\right)\right]
\end{aligned}
$$

where $O$ is either $O^{1 N}$ from Eq. (14) or $O^{2 N}$ from Eq. (19), $E=\frac{\mathbf{p}^{2}}{m}$ is the relative energy of the final $2 \mathrm{~N}$ state and

$$
[\mathbf{f}(|\mathbf{p}|)]=\int_{0}^{2 \pi} d \phi \int_{-1}^{1} d x\left[\left\langle\mathbf{p}^{\prime}|t(E)| \mathbf{p}\right\rangle\right]\left[O\left(\mathbf{p}, \mathbf{P}^{\prime}\right)\right]
$$

since

$$
\mathbf{p}=|\mathbf{p}|\left(\sqrt{1-x^{2}} \cos \phi, \sqrt{1-x^{2}} \sin \phi, x\right) .
$$

The integral (30) with the cut-off value $\bar{p}$ can be easily calculated numerically. In the next section we show how to prepare its component $\left[O\left(\mathbf{p}, \mathbf{P}^{\prime}\right)\right]$.

\section{Explicit Representation of the Spin-Isospin Operators}

Now that the form of expressions in (7) has been established, it remains to find a way to represent operators and states in the isospin-spin space. Once an appropriate matrix representation is found, numerical calculations can be constructed using Eqs. (14), (19) and (30) by simple substitutions and matrix multiplications.

Our choice for the 16-dimensional basis of the two nucleon isospin-spin state space (the deuteron in the initial state, the proton and the neutron in the final state) is the set of vector states $\left\{\left|e_{i}\right\rangle\right\}(i=1,2, \ldots, 16)$ :

$$
\left|e_{i}\right\rangle=\left(\left|m_{1}^{\text {isospin }}(i)\right\rangle \otimes\left|m_{2}^{\text {isospin }}(i)\right\rangle\right) \otimes\left(\left|m_{1}^{\text {spin }}(i)\right\rangle \otimes\left|m_{2}^{\text {spin }}(i)\right\rangle\right)
$$

where $m_{j}^{\text {spin(isospin) }}$ are the spin (isospin) projections of nucleon $j$ and the corresponding quantum numbers are given in Table 1.

Any operator or state in this space can be constructed using the notion of the Kronecker product (KP). The Mathematica ${ }^{\circledR}$ [1] symbolic programming software contains definitions for the KP, which makes translating expressions for isospin-spin operators a simple task. However, it is important to remember that the order of operators in the KP must be preserved; Table 1 can serve as a reference to keep consistence with this paper. In practice all the $\left|e_{i}\right\rangle$ states can be represented explicitly using the KP and the well known spinor forms:

$$
\left|\frac{1}{2} \frac{1}{2}\right\rangle \leftrightarrow\left(\begin{array}{l}
1 \\
0
\end{array}\right), \quad\left|\frac{1}{2}-\frac{1}{2}\right\rangle \leftrightarrow\left(\begin{array}{l}
0 \\
1
\end{array}\right) .
$$

In particular, the deuteron isospin-spin states appearing in Eq. (9) can be constructed using the Clebsch-Gordan coefficients built in Mathematica ${ }^{\circledR}$. For example $[|00\rangle \otimes \chi(1)]$ becomes:

$$
\left(0,0,0,0, \frac{1}{\sqrt{2}}, 0,0,0,-\frac{1}{\sqrt{2}}, 0,0,0,0,0,0,0\right)
$$


Table 1 Reference quantum numbers for our KP states

\begin{tabular}{lllll}
\hline$i$ & $m_{1}^{\text {isospin }}(i)$ & $m_{2}^{\text {isospin }}(i)$ & $m_{1}^{\text {spin }}(i)$ & $m_{2}^{\text {spin }}(i)$ \\
\hline 1 & $\frac{1}{2}$ & $\frac{1}{2}$ & $\frac{1}{2}$ & $\frac{1}{2}$ \\
2 & $\frac{1}{2}$ & $\frac{1}{2}$ & $\frac{1}{2}$ & $-\frac{1}{2}$ \\
3 & $\frac{1}{2}$ & $-\frac{1}{2}$ & $\frac{1}{2}$ & $\frac{1}{2}$ \\
4 & $\frac{1}{2}$ & $-\frac{1}{2}$ & $\frac{1}{2}$ & $\frac{1}{2}$ \\
5 & $\frac{1}{2}$ & $\frac{1}{2}$ & $-\frac{1}{2}$ & $-\frac{1}{2}$ \\
6 & $\frac{1}{2}$ & $-\frac{1}{2}$ & $-\frac{1}{2}$ & $\frac{1}{2}$ \\
7 & $\frac{1}{2}$ & $-\frac{1}{2}$ & $-\frac{1}{2}$ & $-\frac{1}{2}$ \\
8 & $\frac{1}{2}$ & $\frac{1}{2}$ & $-\frac{1}{2}$ & $\frac{1}{2}$ \\
9 & $\frac{1}{2}$ & $\frac{1}{2}$ & $\frac{1}{2}$ & $-\frac{1}{2}$ \\
10 & $-\frac{1}{2}$ & $-\frac{1}{2}$ & $\frac{1}{2}$ & $\frac{1}{2}$ \\
11 & $-\frac{1}{2}$ & $-\frac{1}{2}$ & $\frac{1}{2}$ & $-\frac{1}{2}$ \\
12 & $-\frac{1}{2}$ & $\frac{1}{2}$ & $\frac{1}{2}$ & $\frac{1}{2}$ \\
12 & $-\frac{1}{2}$ & $\frac{1}{2}$ & $-\frac{1}{2}$ & $-\frac{1}{2}$ \\
14 & $-\frac{1}{2}$ & $-\frac{1}{2}$ & $-\frac{1}{2}$ & $\frac{1}{2}$ \\
15 & $-\frac{1}{2}$ & $-\frac{1}{2}$ & $-\frac{1}{2}$ & $-\frac{1}{2}$. \\
16 & $-\frac{1}{2}$ & & $-\frac{1}{2}$ & \\
\hline
\end{tabular}

Similarly, any isospin or spin operator can be represented using the standard Pauli matrices:

$$
\sigma^{x}=\left(\begin{array}{ll}
0 & 1 \\
1 & 0
\end{array}\right), \quad \sigma^{y}=\left(\begin{array}{cc}
0 & -i \\
i & 0
\end{array}\right), \quad \sigma^{z}=\left(\begin{array}{cc}
1 & 0 \\
0 & -1
\end{array}\right) .
$$

For example, the deuteron operators $\left[B_{1}(\mathbf{p})\right]$ and $\left[B_{2}(\mathbf{p})\right]$ with $\mathbf{p}=\left(p_{x}, p_{y}, p_{z}\right)$ from Eq. (9) have a form simple enough to have their matrix representation written out in full. $\left[B_{1}\right]$ is simply a $16 \times 16$ identity matrix. $\left[B_{2}\right]$ has a block diagonal form:

$$
\left(\begin{array}{llll}
B & 0 & 0 & 0 \\
0 & B & 0 & 0 \\
0 & 0 & B & 0 \\
0 & 0 & 0 & B
\end{array}\right),
$$

where $B$ is a $4 \times 4$ matrix:

$$
\left(\begin{array}{cccc}
\frac{1}{3}\left(-p_{x}^{2}-p_{y}^{2}-p_{z}^{2}\right)+p_{z}^{2} & p_{z}\left(p_{x}-i p_{y}\right) & p_{z}\left(p_{x}-i p_{y}\right) & \left(p_{x}-i p_{y}\right)^{2} \\
p_{z}\left(p_{x}+i p_{y}\right) & \frac{1}{3}\left(-p_{x}^{2}-p_{y}^{2}-p_{z}^{2}\right)-p_{z}^{2} & \left(p_{x}-i p_{y}\right)\left(p_{x}+i p_{y}\right) & p_{z}\left(-\left(p_{x}-i p_{y}\right)\right) \\
p_{z}\left(p_{x}+i p_{y}\right) & \left(p_{x}-i p_{y}\right)\left(p_{x}+i p_{y}\right) & \frac{1}{3}\left(-p_{x}^{2}-p_{y}^{2}-p_{z}^{2}\right)-p_{z}^{2} & p_{z}\left(-\left(p_{x}-i p_{y}\right)\right) \\
\left(p_{x}+i p_{y}\right)^{2} & p_{z}\left(-\left(p_{x}+i p_{y}\right)\right) & p_{z}\left(-\left(p_{x}+i p_{y}\right)\right) & \frac{1}{3}\left(-p_{x}^{2}-p_{y}^{2}-p_{z}^{2}\right)+p_{z}^{2}
\end{array}\right) .
$$

We consider now in detail matrix representations of the different parts of the $2 \mathrm{~N}$ current operator. It consists of the single-nucleon and $2 \mathrm{~N}$ operators. In this paper we assume that its single-nucleon part comprises the standard nonrelativistic charge density $\left(\mathbf{j}^{0}\right)$ as well as the convection $\left(\mathbf{j}^{\mathrm{conv}}\right)$ and $\operatorname{spin}\left(\mathbf{j}^{\mathbf{s p i n}}\right)$ current operators. The following expressions are matrix elements in the momentum space but operators in the isospin and spin spaces for the single nucleon operators of nucleon 2 :

$$
\left\langle\mathbf{p}_{2}^{\prime}\left|\mathbf{j}^{0}(2)\right| \mathbf{p}_{2}\right\rangle=\left(\frac{1}{2} G_{E}^{p}\left(1+\tau^{z}(2)\right)+\frac{1}{2} G_{E}^{n}\left(1-\tau^{z}(2)\right)\right),
$$




$$
\begin{aligned}
\left\langle\mathbf{p}_{2}^{\prime}\left|\mathbf{j}^{\text {conv }}(2)\right| \mathbf{p}_{2}\right\rangle= & \left(\frac{1}{2} G_{E}^{p}\left(1+\tau^{z}(2)\right)+\frac{1}{2} G_{E}^{n}\left(1-\tau^{z}(2)\right)\right) \\
& \times \frac{\mathbf{p}_{2}^{\prime}+\mathbf{p}_{2}}{2 m}, \\
\left\langle\mathbf{p}_{2}^{\prime}\left|\mathbf{j}^{\text {spin }}(2)\right| \mathbf{p}_{2}\right\rangle= & \left(\frac{1}{2} G_{M}^{p}\left(\mathbf{1}+\tau^{z}(2)\right)+\frac{1}{2} G_{M}^{n}\left(\mathbf{1}-\tau^{z}(2)\right)\right) \\
& \times \frac{i \boldsymbol{\sigma}(2) \times\left(\mathbf{p}_{2}^{\prime}-\mathbf{p}_{2}\right)}{2 m} .
\end{aligned}
$$

In the above expressions $G_{E}^{p}\left(G_{E}^{n}\right)$ and $G_{M}^{p}\left(G_{M}^{n}\right)$ are the proton (neutron) electric and magnetic form factors. The isospin projection operator, acting in the $2 \mathrm{~N}$ isospin space, $\Pi_{E, M}(2) \equiv \frac{1}{2} G_{E, M}^{p}\left(\mathbf{1}+\tau^{z}(2)\right)+$ $\frac{1}{2} G_{E, M}^{n}\left(\mathbf{1}-\tau^{z}(2)\right)$, is represented by a diagonal $4 \times 4$ matrix:

$$
\Pi_{E, M}(2) \leftrightarrow\left(\begin{array}{cccc}
G_{E, M}^{p} & 0 & 0 & 0 \\
0 & G_{E, M}^{n} & 0 & 0 \\
0 & 0 & G_{E, M}^{p} & 0 \\
0 & 0 & 0 & G_{E, M}^{n}
\end{array}\right)
$$

The spin part of the charge density corresponds just to the unit $4 \times 4$ matrix and for every component of the convection current we obtain a matrix proportional to the unit matrix. Only the spin part of the spin current operator is not diagonal. The matrices corresponding to $\left[j\left(2, \mathbf{P}^{\prime},-2 \mathbf{p}^{\prime}\right)\right]$, from Eq. (14) for the charge density $(R(2))$, spherical +1 component of the convection current $\left((C(2))_{+1}\right)$ and spin current $\left((S(2))_{+1}\right)$ are obtained as

$$
\begin{aligned}
R(2) & =\Pi_{E}(2) \otimes\left(\begin{array}{cccc}
1 & 0 & 0 & 0 \\
0 & 1 & 0 & 0 \\
0 & 0 & 1 & 0 \\
0 & 0 & 0 & 1
\end{array}\right), \\
(C(2))_{+1} & =\Pi_{E}(2) \otimes\left(\begin{array}{cccc}
-\frac{p_{x}+i p_{y}}{\sqrt{2} m} & 0 & 0 & 0 \\
0 & -\frac{p_{x}+i p_{y}}{\sqrt{2} m} & 0 & 0 \\
0 & 0 & -\frac{p_{x}+i p_{y}}{\sqrt{2} m} & 0 \\
0 & 0 & 0 & -\frac{p_{x}+i p_{y}}{\sqrt{2} m}
\end{array}\right), \\
(S(2))_{+1} & =\Pi_{M}(2) \otimes\left(\begin{array}{cccc}
0 & -\frac{Q}{\sqrt{2} m} & 0 & 0 \\
0 & 0 & 0 & 0 \\
0 & 0 & 0 & -\frac{Q}{\sqrt{2} m} \\
0 & 0 & 0 & 0
\end{array}\right),
\end{aligned}
$$

where in the convection current $\mathbf{p}_{2}^{\prime}+\mathbf{p}_{2}$ has been replaced by $-2 \mathbf{p}$ and in the spin current $\mathbf{P}^{\prime}=\mathbf{p}_{2}^{\prime}-\mathbf{p}_{2}=\mathbf{Q} \| \hat{\mathbf{z}}$.

In the $2 \mathrm{~N}$ part of the current operator we consider for simplicity only the leading one-pion-exchange current operator in the chiral effective field theory representation. The corresponding expression in the momentum space reads:

$$
\begin{aligned}
& \left\langle\mathbf{p}_{1}^{\prime} \mathbf{p}_{2}^{\prime}\left|\mathbf{j}^{\mathbf{1} \pi}(1,2)\right| \mathbf{p}_{1} \mathbf{p}_{2}\right\rangle=i(\boldsymbol{\tau}(1) \times \boldsymbol{\tau}(2))^{z} \\
& \quad \times\left(\left(\frac{g_{A}}{2 F_{\pi}}\right)^{2} \frac{\boldsymbol{\sigma}(2) \cdot \mathbf{q}_{2}}{\mathbf{q}_{2}^{2}+m_{\pi}^{2}} \boldsymbol{\sigma}(1)-\left(\frac{g_{A}}{2 F_{\pi}}\right)^{2} \frac{\boldsymbol{\sigma}(1) \cdot \mathbf{q}_{1}}{\mathbf{q}_{1}^{2}+m_{\pi}^{2}} \boldsymbol{\sigma}(2)\right. \\
& \left.\quad+\left(\frac{g_{A}}{2 F_{\pi}}\right)^{2} \frac{\boldsymbol{\sigma}(1) \cdot \mathbf{q}_{1}}{\mathbf{q}_{1}^{2}+m_{\pi}^{2}} \frac{\boldsymbol{\sigma}(2) \cdot \mathbf{q}_{2}}{\mathbf{q}_{2}^{2}+m_{\pi}^{2}}\left(\mathbf{q}_{1}-\mathbf{q}_{2}\right)\right),
\end{aligned}
$$


where $\mathbf{q}_{1} \equiv \mathbf{p}_{i}^{\prime}-\mathbf{p}_{i}$, and $m_{\pi}, F_{\pi}$ and $g_{A}$ denote the pion mass, the pion decay constant and the nucleon axial coupling constant, respectively. Its isospin part, $\Pi(1,2) \equiv i(\boldsymbol{\tau}(1) \times \boldsymbol{\tau}(2))^{z}$, is again very simple

$$
\Pi(1,2) \leftrightarrow\left(\begin{array}{cccc}
0 & 0 & 0 & 0 \\
0 & 0 & -2 & 0 \\
0 & 2 & 0 & 0 \\
0 & 0 & 0 & 0
\end{array}\right) .
$$

The spin part of the one-pion-exchange current operator $(\mathbf{J})$ has a more complicated form. We choose again the spherical +1 component but are forced to show the individual matrix elements, $J_{k l}$ :

$$
\begin{aligned}
& J_{11}=\frac{q_{1 z} q_{2 z} v_{12}\left(-q_{1 x}-i q_{1 y}+q_{2 x}+i q_{2 y}\right)}{\sqrt{2}}, \\
& J_{12}=\frac{-2 q_{1 z} v_{1}-q_{1 z} v_{12}\left(q_{2 x}-i q_{2 y}\right)\left(q_{1 x}+i\left(q_{1 y}+i q_{2 x}-q_{2 y}\right)\right)}{\sqrt{2}}, \\
& J_{13}=\frac{q_{2 z}\left(2 v_{2}-v_{12}\left(q_{1 x}-i q_{1 y}\right)\left(q_{1 x}+i\left(q_{1 y}+i q_{2 x}-q_{2 y}\right)\right)\right)}{\sqrt{2}}, \\
& J_{14}=\frac{2 v_{2}\left(q_{2 x}-i q_{2 y}\right)-\left(q_{1 x}-i q_{1 y}\right)\left(2 v_{1}+v_{12}\left(q_{2 x}-i q_{2 y}\right)\left(q_{1 x}+i\left(q_{1 y}+i q_{2 x}-q_{2 y}\right)\right)\right)}{\sqrt{2}}, \\
& J_{21}=\frac{q_{1 z} v_{12}\left(q_{2 x}+i q_{2 y}\right)\left(-q_{1 x}-i q_{1 y}+q_{2 x}+i q_{2 y}\right)}{\sqrt{2}}, \\
& J_{22}=\frac{q_{1 z} q_{2 z} v_{12}\left(q_{1 x}+i\left(q_{1 y}+i q_{2 x}-q_{2 y}\right)\right)}{\sqrt{2}}, \\
& J_{23}=\frac{\left(q_{2 x}+i q_{2 y}\right)\left(2 v_{2}-v_{12}\left(q_{1 x}-i q_{1 y}\right)\left(q_{1 x}+i\left(q_{1 y}+i q_{2 x}-q_{2 y}\right)\right)\right)}{\sqrt{2}}, \\
& J_{24}=\frac{-2 q_{2 z} v_{2}+q_{2 z} v_{12}\left(q_{1 x}-i q_{1 y}\right)\left(q_{1 x}+i\left(q_{1 y}+i q_{2 x}-q_{2 y}\right)\right)}{\sqrt{2}}, \\
& J_{31}=-\frac{q_{2 z} v_{12}\left(q_{1 x}+i q_{1 y}\right)\left(q_{1 x}+i\left(q_{1 y}+i q_{2 x}-q_{2 y}\right)\right)}{\sqrt{2}}, \\
& J_{32}=-\frac{\left(q_{1 x}+i q_{1 y}\right)\left(2 v_{1}+v_{12}\left(q_{2 x}-i q_{2 y}\right)\left(q_{1 x}+i\left(q_{1 y}+i q_{2 x}-q_{2 y}\right)\right)\right)}{\sqrt{2}}, \\
& J_{33}=\frac{q_{1 z} q_{2 z} v_{12}\left(q_{1 x}+i\left(q_{1 y}+i q_{2 x}-q_{2 y}\right)\right)}{\sqrt{2}}, \\
& J_{34}=\frac{2 q_{1 z} v_{1}+q_{1 z} v_{12}\left(q_{2 x}-i q_{2 y}\right)\left(q_{1 x}+i\left(q_{1 y}+i q_{2 x}-q_{2 y}\right)\right)}{\sqrt{2}}, \\
& J_{41}=-\frac{v_{12}\left(q_{1 x}+i q_{1 y}\right)\left(q_{2 x}+i q_{2 y}\right)\left(q_{1 x}+i\left(q_{1 y}+i q_{2 x}-q_{2 y}\right)\right)}{\sqrt{2}}, \\
& J_{42}=\frac{q_{2 z} v_{12}\left(q_{1 x}+i q_{1 y}\right)\left(q_{1 x}+i\left(q_{1 y}+i q_{2 x}-q_{2 y}\right)\right)}{\sqrt{2}}, \\
& J_{43}=\frac{q_{1 z} v_{12}\left(q_{2 x}+i q_{2 y}\right)\left(q_{1 x}+i\left(q_{1 y}+i q_{2 x}-q_{2 y}\right)\right)}{\sqrt{2}}, \\
& J_{44}=\frac{q_{1 z} q_{2 z} v_{12}\left(-q_{1 x}-i q_{1 y}+q_{2 x}+i q_{2 y}\right)}{\sqrt{2}},
\end{aligned}
$$

where

and

$$
v_{i}=\frac{g_{A}^{2}}{\left(2 F_{\pi}\right)^{2}\left(\mathbf{q}_{i}^{2}+m_{\pi}^{2}\right)}
$$

$$
v_{12}=\frac{g_{A}^{2}}{\left(2 F_{\pi}\right)^{2}\left(\mathbf{q}_{1}^{2}+m_{\pi}^{2}\right)\left(\mathbf{q}_{2}^{2}+m_{\pi}^{2}\right)} .
$$




\begin{tabular}{|c|c|c|c|c|c|c|c|c|c|c|c|c|c|}
\hline$(0$ & $\begin{array}{ll}0 & 0\end{array}$ & 00 & 0 & 0 & 0 & 0 & 0 & 0 & 0 & 0 & $\begin{array}{ll}0 & 0\end{array}$ & 0 & $0)$ \\
\hline 0 & $\begin{array}{ll}0 & 0\end{array}$ & $\begin{array}{ll}0 & 0\end{array}$ & 0 & 0 & 0 & 0 & 0 & 0 & 0 & 0 & $\begin{array}{ll}0 & 0\end{array}$ & 0 & 0 \\
\hline 0 & $\begin{array}{ll}0 & 0\end{array}$ & 00 & 0 & 0 & 0 & 0 & 0 & 0 & 0 & 0 & $\begin{array}{ll}0 & 0\end{array}$ & 0 & 0 \\
\hline 0 & $\begin{array}{ll}0 & 0\end{array}$ & $\begin{array}{ll}0 & 0\end{array}$ & 0 & 0 & 0 & 0 & 0 & 0 & 0 & 0 & $\begin{array}{ll}0 & 0\end{array}$ & 0 & 0 \\
\hline 0 & $\begin{array}{ll}0 & 0\end{array}$ & 0 & 0 & $-2 i \mathrm{q}_{2 \mathrm{x}}-2 \mathrm{q}_{2 \mathrm{y}}$ & $-2 i \mathrm{q}_{1 \mathrm{x}}-2 \mathrm{q}_{1 \mathrm{y}}$ & 0 & 0 & 0 & 0 & 0 & $\begin{array}{ll}0 & 0\end{array}$ & 0 & 0 \\
\hline 0 & $\begin{array}{ll}0 & 0\end{array}$ & 0 & $2 i \mathrm{q}_{2 \mathrm{x}}-2 \mathrm{q}_{2 \mathrm{y}}$ & 0 & 0 & $-2 i \mathrm{q}_{1 \mathrm{x}}-2 \mathrm{q}_{1 \mathrm{y}}$ & 0 & 0 & 0 & 0 & $\begin{array}{ll}0 & 0\end{array}$ & 0 & 0 \\
\hline 0 & $\begin{array}{ll}0 & 0\end{array}$ & 00 & $2 i \mathrm{q}_{1 \mathrm{x}}-2 \mathrm{q}_{1 \mathrm{y}}$ & 0 & 0 & $-2 i \mathrm{q}_{2 \mathrm{x}}-2 \mathrm{q}_{2 \mathrm{y}}$ & 0 & 0 & 0 & 0 & $\begin{array}{ll}0 & 0\end{array}$ & 0 & 0 \\
\hline 0 & $\begin{array}{ll}0 & 0\end{array}$ & 00 & 0 & $2 i \mathrm{q}_{1 \mathrm{x}}-2 \mathrm{q}_{\mathrm{ly}}$ & $2 i \mathrm{q}_{2 \mathrm{x}}-2 \mathrm{q}_{2 \mathrm{y}}$ & 0 & 0 & 0 & 0 & 0 & $\begin{array}{ll}0 & 0\end{array}$ & 0 & 0 \\
\hline 0 & $\begin{array}{ll}0 & 0\end{array}$ & 0 & 0 & 0 & 0 & 0 & 0 & $2\left(i \mathrm{q}_{2 \mathrm{x}}+\mathrm{q}_{2 \mathrm{y}}\right)$ & $2\left(i \mathrm{q}_{1 \mathrm{x}}+\mathrm{q}_{1 \mathrm{y}}\right)$ & 0 & $\begin{array}{ll}0 & 0\end{array}$ & 0 & 0 \\
\hline 0 & $\begin{array}{ll}0 & 0\end{array}$ & 00 & 0 & 0 & 0 & 0 & $2\left(-i \mathrm{q}_{2 \mathrm{x}}+\mathrm{q}_{2 \mathrm{y}}\right)$ & 0 & 0 & $2\left(i \mathrm{q}_{1 \mathrm{x}}+\mathrm{q}_{1 \mathrm{y}}\right)$ & $\begin{array}{ll}0 & 0\end{array}$ & 0 & 0 \\
\hline 0 & $\begin{array}{ll}0 & 0\end{array}$ & 00 & 0 & 0 & 0 & 0 & $2\left(-i \mathrm{q}_{1 \mathrm{x}}+\mathrm{q}_{1 \mathrm{y}}\right)$ & 0 & 0 & $2\left(i \mathrm{q}_{2 \mathrm{x}}+\mathrm{q}_{2 \mathrm{y}}\right)$ & $\begin{array}{ll}0 & 0\end{array}$ & 0 & 0 \\
\hline 0 & $\begin{array}{ll}0 & 0\end{array}$ & 0 & 0 & 0 & 0 & 0 & 0 & $2\left(-i \mathrm{q}_{1 \mathrm{x}}+\mathrm{q}_{1 \mathrm{y}}\right)$ & $2\left(-i q_{2 x}+q_{2 y}\right)$ & 0 & $\begin{array}{ll}0 & 0\end{array}$ & 0 & 0 \\
\hline 0 & $\begin{array}{ll}0 & 0\end{array}$ & 00 & 0 & 0 & 0 & 0 & 0 & 0 & 0 & 0 & $\begin{array}{ll}0 & 0\end{array}$ & 0 & 0 \\
\hline 0 & $\begin{array}{ll}0 & 0\end{array}$ & 00 & 0 & 0 & 0 & 0 & 0 & 0 & 0 & 0 & $\begin{array}{ll}0 & 0\end{array}$ & 0 & 0 \\
\hline 0 & $\begin{array}{ll}0 & 0\end{array}$ & 00 & 0 & 0 & 0 & 0 & 0 & 0 & 0 & 0 & $\begin{array}{lll}0 & 0\end{array}$ & 0 & 0 \\
\hline 0 & $\begin{array}{ll}0 & 0\end{array}$ & 00 & 0 & 0 & 0 & 0 & 0 & 0 & 0 & 0 & $\begin{array}{ll}0 & 0\end{array}$ & 0 & $0)$ \\
\hline
\end{tabular}

Fig. $1\left[T_{2} O_{5}^{3}\right]\left(\mathbf{p}_{1}^{\prime}-\mathbf{p}_{1}=\left(q_{1 x}, q_{1 y}, q_{1 z}\right), \mathbf{p}_{2}^{\prime}-\mathbf{p}_{2}=\left(q_{2 x}, q_{2 y}, q_{2 z}\right)\right)$ with $T_{2}=\boldsymbol{\tau}^{z}(1)-\boldsymbol{\tau} z(2)$ and $O_{5}=\left(\mathbf{q}_{1} \times \boldsymbol{\sigma}(1)\right)+\left(\mathbf{q}_{2} \times\right.$ $\boldsymbol{\sigma}(2)) . \boldsymbol{\tau}(i)(\boldsymbol{\sigma}(i))$ is the $2 \mathrm{~N}$ isospin (spin) vector operator acting in the space of nucleon $i$
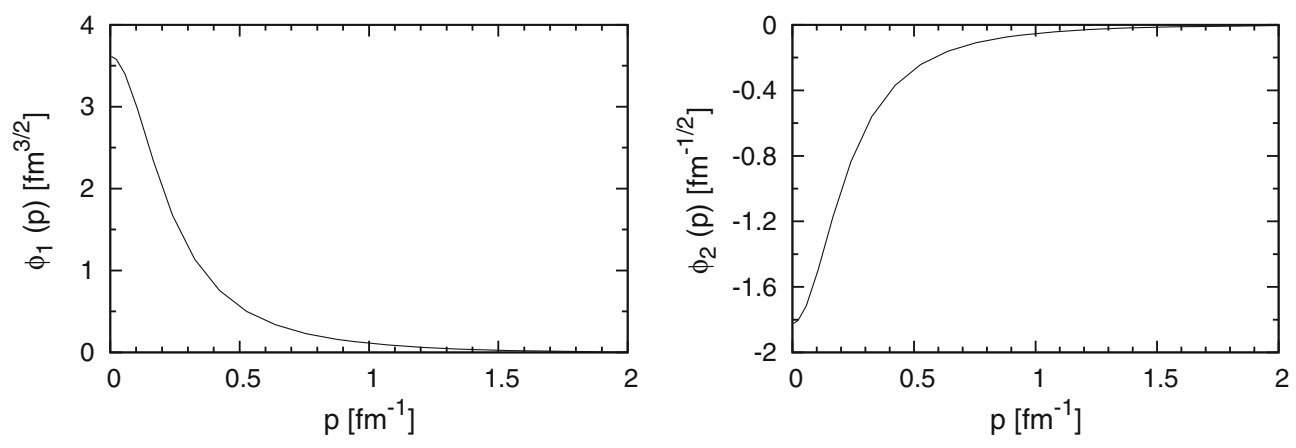

Fig. 2 The $\phi_{1}(p)$ (left) and $\phi_{2}(p)$ (right) expansion function in the operator form of the deuteron as a function of the magnitude of the relative momentum $p$ for the considered chiral NNLO potential
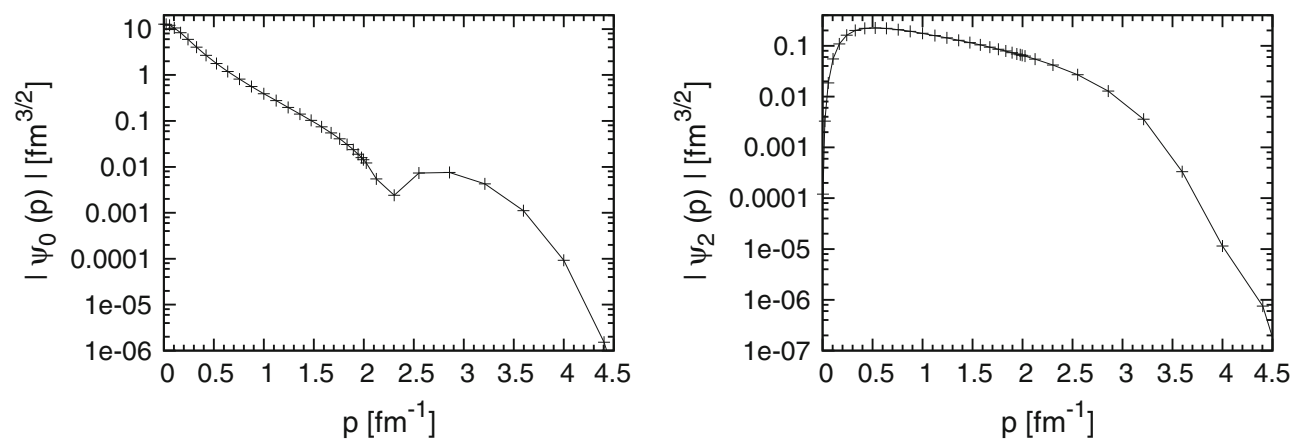

Fig. 3 The $s$-wave (left) and $d$-wave (right) component of the deuteron wave function as a function of the magnitude of the relative momentum $p$ for the considered chiral NNLO potential. Crosses represent results obtained using the operator approach and solid lines are directly from the standard partial wave decomposition

In the laboratory frame the matrix $\left(J_{1 \pi}(1,2)\right)_{+1}$ corresponding to

$$
\left[j\left(1,2, \frac{1}{2} \mathbf{P}^{\prime}+\mathbf{p}^{\prime}-\mathbf{p}^{\prime \prime}, \frac{1}{2} \mathbf{P}^{\prime}-\mathbf{p}^{\prime}+\mathbf{p}^{\prime \prime}\right)\right]
$$

reads

$$
\left(J_{1 \pi}\right)_{+1}=\Pi(1,2) \otimes J
$$

where now $\mathbf{q}_{1}=\frac{1}{2} \mathbf{P}^{\prime}+\mathbf{p}^{\prime}-\mathbf{p}^{\prime \prime}$ and $\mathbf{q}_{2}=\frac{1}{2} \mathbf{P}^{\prime}-\mathbf{p}^{\prime}+\mathbf{p}^{\prime \prime}$.

Matrices corresponding to the isospin-spin operators appearing in (16) and (17) can be constructed in the same way. An explicit example of such $16 \times 16$ matrix is given below in Fig. 1 . 

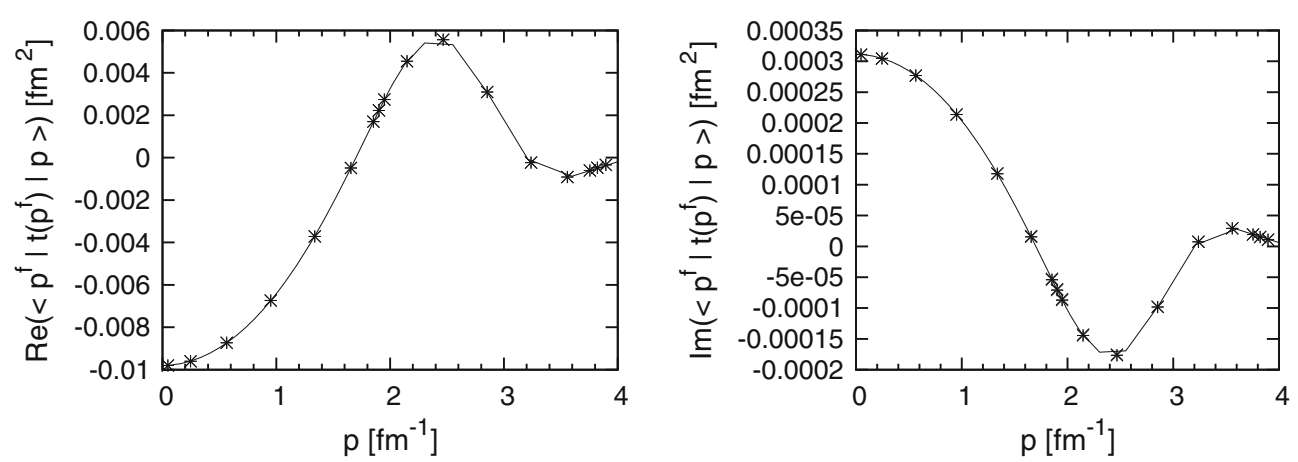

Fig. 4 The real (left) and imaginary (right) parts of the half-shell ${ }^{1} S_{0}$ t-matrix as a function of the initial momentum $p$ for $p^{\mathrm{f}} \approx$ $1.9 \mathrm{fm}^{-1}$. Points represent predictions obtained by a projection from the 3D results. Solid lines represent direct solutions of LSE in the standard partial wave decomposition
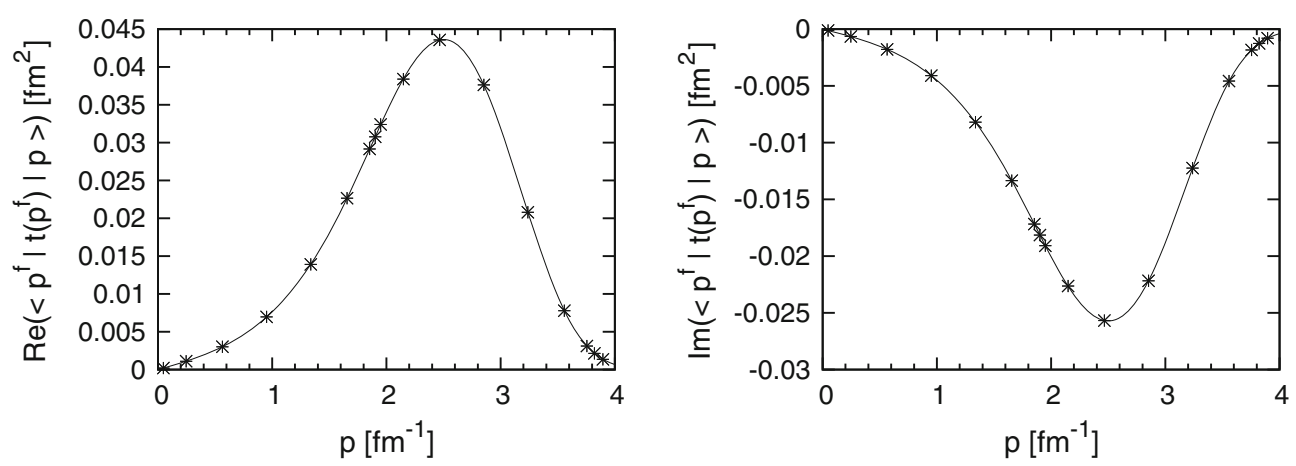

Fig. 5 The same as in Fig. 4 for the half-shell ${ }^{3} P_{0}$ t-matrix
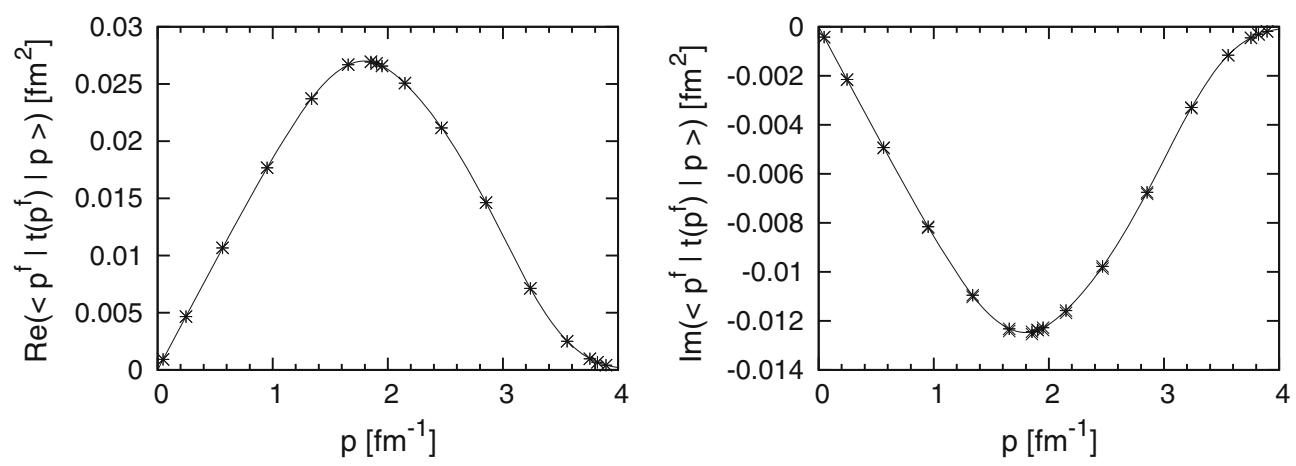

Fig. 6 The same as in Fig. 4 for the half-shell ${ }^{1} P_{1}$ t-matrix

\section{Results}

In the following we will present results obtained using a chiral NNLO potential [13] with $\Lambda=550 \mathrm{MeV} / \mathrm{c}$ and $\tilde{\Lambda}=600 \mathrm{MeV} / \mathrm{c}$. The operator form of such a potential was briefly described in Appendix C of Ref. [7], where also an example set of necessary parameters was given for its neutron-proton version. The same parameters will also be used in the present paper.

There are three basic ingredients in our calculations: the deuteron wave function, the $2 \mathrm{~N}$ t-matrix and the $2 \mathrm{~N}$ current operator. Before we show selected observables for the ${ }^{2} \mathrm{H}\left(e, e^{\prime} p\right) n$ reaction, we will describe our numerical performance and the way we verify the quality of our calculations.

As described in Ref. [7] and Eq. (9), the deuteron in the operator form is represented by two functions $\phi_{1}(p)$ and $\phi_{2}(p)$. The corresponding Schrödinger equation for $\phi_{1}(p)$ and $\phi_{2}(p)$ can be rewritten as an eigenvalue problem, which is of the same type and dimension as the one solved for the deuteron wave function in the 

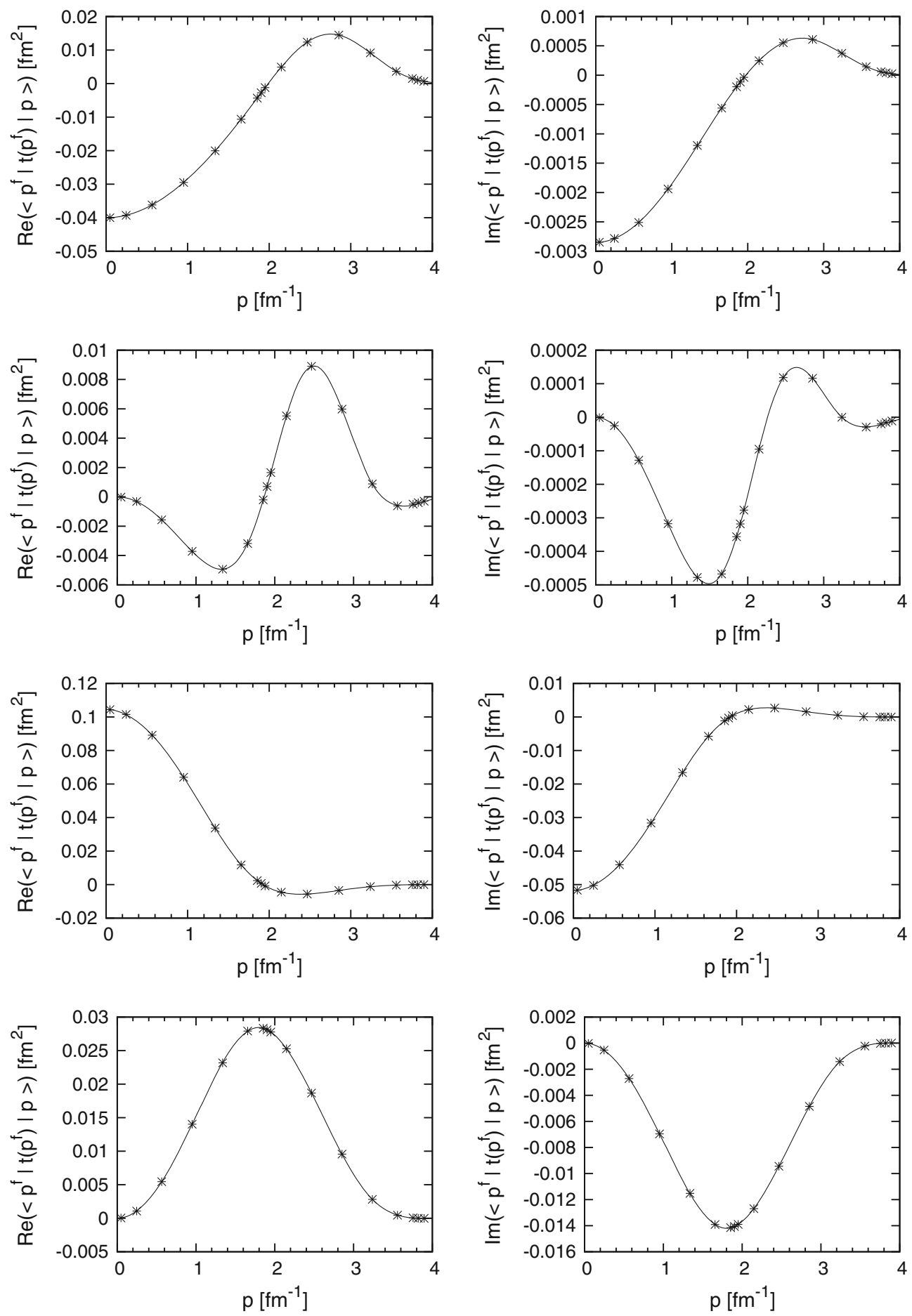

Fig. 7 The same as in Fig. 4 for the half-shell ${ }^{3} S_{1}-{ }^{3} D_{1}$ t-matrix. Rows show different $l$ and $l^{\prime}$ cases (from top to bottom): $(l=0$, $\left.l^{\prime}=0\right),\left(l=2, l^{\prime}=0\right),\left(l=0, l^{\prime}=2\right)$ and $\left(l=2, l^{\prime}=2\right)$

standard partial wave representation, where one deals with the $s$ - and $d$-components, $\psi_{0}(p)$ and $\psi_{2}(p)$. The connection between the solutions is very simple [6]

$$
\psi_{0}(p)=\sqrt{4 \pi} \phi_{1}(p), \quad \psi_{2}(p)=\frac{4 \sqrt{2} p^{2}}{3} \phi_{2}(p)
$$

and can be used to check the numerical performance. 
Table 2 The six electron kinematics considered in the paper for the exclusive ${ }^{2} \mathrm{H}\left(e, e^{\prime} p\right) n$ process

\begin{tabular}{|c|c|c|c|c|c|c|}
\hline & $\begin{array}{l}E_{e} \\
\mathrm{MeV}\end{array}$ & $\begin{array}{l}\theta_{e} \\
\operatorname{deg}\end{array}$ & $\begin{array}{l}E_{e}^{\prime} \\
\mathrm{MeV}\end{array}$ & $\begin{array}{l}p^{\mathrm{f}} \text { of Eq. (6) } \\
\mathrm{MeV} / \mathrm{c}\end{array}$ & $\begin{array}{l}\omega=E_{e}-E_{e}^{\prime} \\
\mathrm{MeV}\end{array}$ & $\begin{array}{l}Q \text { of Eq. (4) } \\
\mathrm{MeV} / \mathrm{c}\end{array}$ \\
\hline$K 1$ & 500 & 6.9 & 490.3 & 78.1 & 9.7 & 60 \\
\hline$K 2$ & 500 & 17.4 & 485.3 & 78.1 & 14.7 & 150 \\
\hline$K 3$ & 500 & 6.1 & 467.0 & 158.7 & 30.0 & 60 \\
\hline$K 4$ & 500 & 36.4 & 447.0 & 158.7 & 53.0 & 300 \\
\hline$K 5$ & 500 & 16.3 & 337.1 & 375.3 & 162.9 & 200 \\
\hline K6 & 500 & 73.7 & 281.2 & 375.3 & 218.8 & 500 \\
\hline
\end{tabular}

The initial electron energy $\left(E_{e}\right)$, the electron scattering angle $\left(\theta_{e}\right)$, the final electron energy $\left(E_{e}^{\prime}\right)$, the final relative nucleon-nucleon momentum $\left(\left|\mathbf{p}^{\mathrm{f}}\right|\right)$, the energy transfer $(\omega)$, and the magnitude of the three-momentum transfer $(Q)$ are given

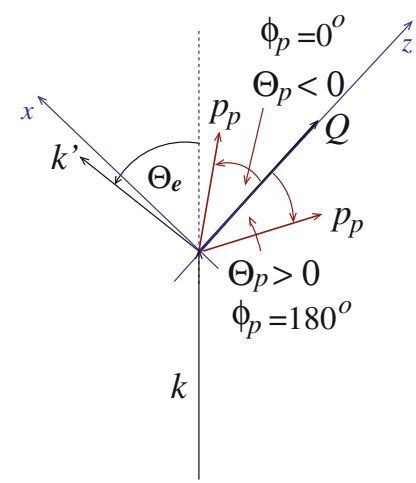

Fig. 8 The kinematics for the exclusive ${ }^{2} \mathrm{H}\left(e, e^{\prime} p\right) n$ process. $\mathbf{k}\left(\mathbf{k}^{\prime}\right)$ is the initial (final) electron momentum. We neglect the electron mass, so $|\mathbf{k}|=E$ and $\left|\mathbf{k}^{\prime}\right|=E^{\prime}$

In Fig. 2 we show directly the $\phi_{1}(p)$ and $\phi_{2}(p)$ functions required for the operator expansion of the deuteron. In Fig. 3 the resulting $s$ - and $d$-wave components in momentum space are compared to the results obtained by firstly decomposing the NN potential into partial waves and then solving the resulting Schrödinger equation in its standard form. The agreement for the two wave function components is perfect for all their significant values.

In Ref. [7] we solved the Lippmann-Schwinger equation (LSE) for the $2 \mathrm{~N} \mathrm{t-matrix} \mathrm{directly} \mathrm{in} \mathrm{three} \mathrm{dimen-}$ sions. At that time we focused mainly on the on-shell behavior of the expansion coefficients $t_{i}\left(p^{\prime}, p, x ; E_{2 N}\right)$, that is we were interested in $t_{i}\left(p_{0}, p_{0}, x ; E_{2 N}=\frac{p_{0}^{2}}{m}\right)$, which are sufficient to calculate the Wolfenstein parameters and the nucleon-nucleon scattering observables. Furthermore, we solved LSE in such a form (Eq. 2.6 from Ref. [7])

$$
\begin{aligned}
& \sum_{j} A_{k j}\left(\mathbf{p}^{\prime}, \mathbf{p}\right) t_{j}^{t m_{t}}\left(\mathbf{p}^{\prime}, \mathbf{p}\right)=\sum_{j} A_{k j}\left(\mathbf{p}^{\prime}, \mathbf{p}\right) v_{j}^{t m_{t}}\left(\mathbf{p}^{\prime}, \mathbf{p}\right) \\
& +\int d^{3} p^{\prime \prime} \sum_{j j^{\prime}} v_{j}^{t m_{t}}\left(\mathbf{p}^{\prime}, \mathbf{p}^{\prime \prime}\right) G_{0}\left(p^{\prime \prime}\right) t_{j^{\prime}}^{t m_{t}}\left(\mathbf{p}^{\prime \prime}, \mathbf{p}\right) B_{k j j^{\prime}}\left(\mathbf{p}^{\prime}, \mathbf{p}^{\prime \prime}, \mathbf{p}\right),
\end{aligned}
$$

that the magnitude of the initial $\mathbf{p}$ momentum could be fixed.

Clearly, for the ${ }^{2} \mathrm{H}\left(e, e^{\prime} p\right) n$ reaction we need a "left" version of Eq. (50), which allows us to find the half-shell t-matrix for a fixed final relative momentum, $\mathbf{p}^{\mathrm{f}}$, given now by the reaction kinematics. The starting point for this new version is equation (20). Repeating the algebra outlined in Ref. [7], we prepared a numerical realization of this "left" version of LSE, leading to the scalar expansion coefficients $t_{i}\left(\mathbf{p}^{\mathrm{f}}, \mathbf{p} ; E_{2 N}=\frac{\left(\mathbf{p}^{\mathrm{f}}\right)^{2}}{m}\right) \equiv$ $t_{i}\left(p^{\mathrm{f}}, p, x ; E_{2 N}=\frac{\left(\mathbf{p}^{\mathrm{f}}\right)^{2}}{m}\right)$, where $x \equiv \hat{p}^{\mathrm{f}} \cdot \hat{p}$. Our numerical scheme was based on the standard LU decomposition from Numerical Recipes [14]. We used (complex versions of) the LUDCMP and LUBKSB subroutines to solve the system of linear equations. In order to achieve a unique and smooth solution also for $p=p^{\mathrm{f}}$, it 

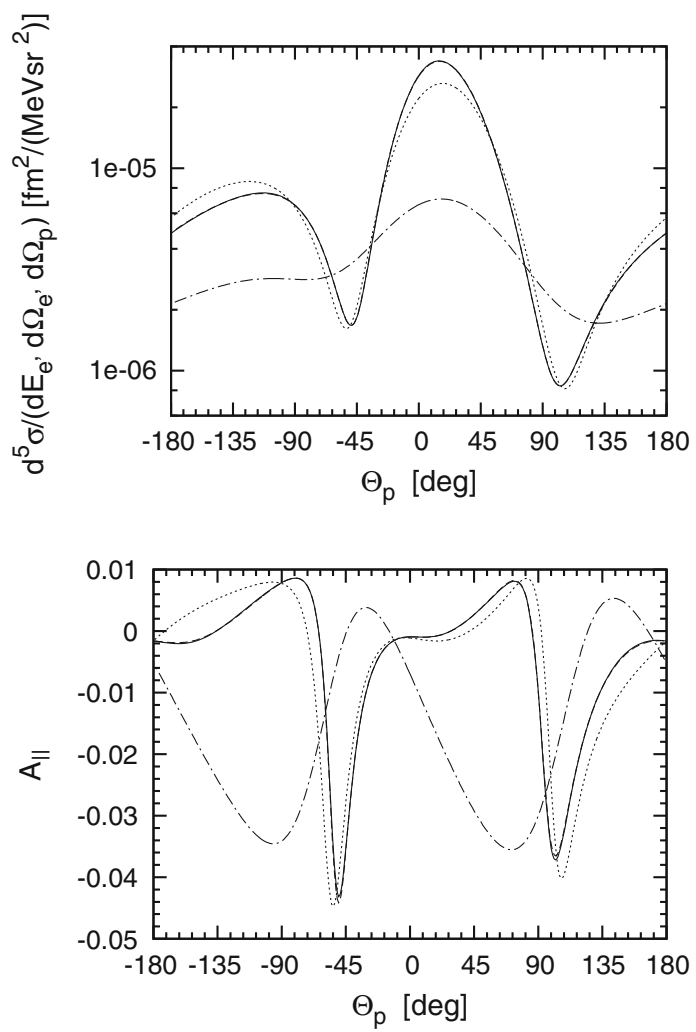

Fig. 9 The unpolarized cross section $d^{5} \sigma /\left(d E_{e^{\prime}} d \Omega_{e^{\prime}} d \Omega_{p}\right)$ and the spin-dependent helicity asymmetry $A_{\|}$as a function of the proton scattering angle $\theta_{p}$ for the $K 3$ electron kinematics from Table 2. Convergence of the full results calculated with a different number of nucleon-nucleon partial waves towards the full 3D prediction (solid line) is shown. Partial wave based results with $j \leq 1$ (dash-dotted line), $j \leq 2$ (dotted line) and $j \leq 4$ (dashed line) are displayed

was sufficient to calculate the average

$$
\begin{aligned}
& t_{i}\left(p^{\mathrm{f}}, p, x ; E_{2 N}=\frac{\left(\mathbf{p}^{\mathrm{f}}\right)^{2}}{m}\right) \\
& \quad=\frac{1}{2}\left(t_{i}\left(p^{\mathrm{f}}-\delta_{p^{\mathrm{f}}}, p, x ; E_{2 N}=\frac{\left(\mathbf{p}^{\mathrm{f}}\right)^{2}}{m}\right)+t_{i}\left(p^{\mathrm{f}}+\delta_{p^{\mathrm{f}}}, p, x ; E_{2 N}=\frac{\left(\mathbf{p}^{\mathrm{f}}\right)^{2}}{m}\right)\right),
\end{aligned}
$$

with $\delta_{p^{\mathrm{f}}} \approx 0.01 \mathrm{fm}^{-1}$.

Actually, this effort turned out to be unnecessary and provided merely an additional check of numerics, since

$$
t_{i}\left(p^{\mathrm{f}}, p, x ; E_{2 N}=\frac{\left(\mathbf{p}^{\mathrm{f}}\right)^{2}}{m}\right)=t_{i}\left(p, p^{\mathrm{f}}, x ; E_{2 N}=\frac{\left(\mathbf{p}^{\mathrm{f}}\right)^{2}}{m}\right)
$$

for the most general rotational, parity and time reversal invariant form of the $\mathrm{NN}$ force. That means that the left coefficients,

$$
t_{i}\left(p^{\mathrm{f}}, p, x ; E_{2 N}=\frac{\left(\mathbf{p}^{\mathrm{f}}\right)^{2}}{m}\right),
$$

can be obtained directly from the "right" version of LSE.

In order to further check our t-matrix coefficients, we used them to calculate the partial wave representation of the t-matrix:

$$
\left\langle p^{\mathrm{f}}\left(l^{\prime} s\right) j\left|t\left(E_{2 N}=\frac{\left(\mathbf{p}^{\mathrm{f}}\right)^{2}}{m}\right)\right| p(l s) j\right\rangle
$$



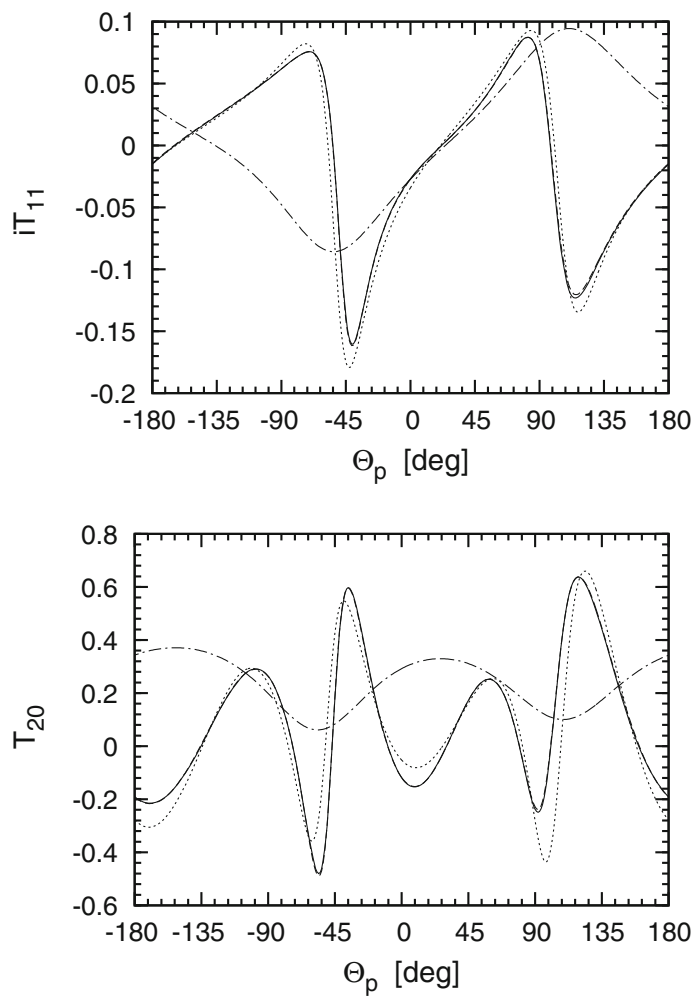

Fig. 10 The same as in Fig. 9 for the deuteron analyzing powers $i T_{11}$ and $T_{20}$
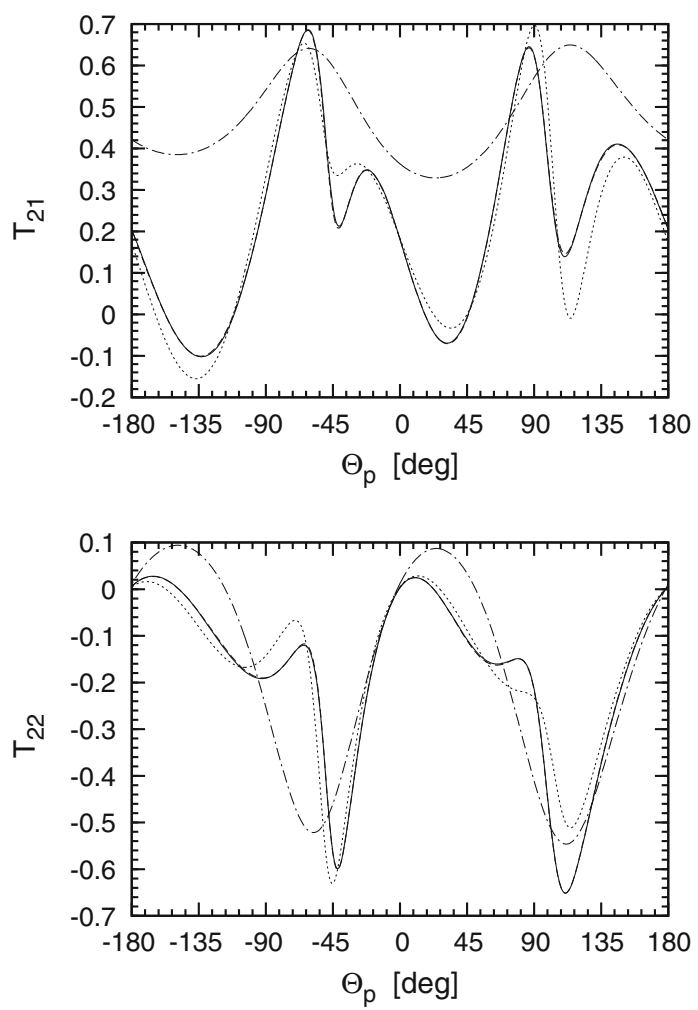

Fig. 11 The same as in Fig. 9 for the deuteron analyzing powers $T_{21}$ and $T_{22}$ 

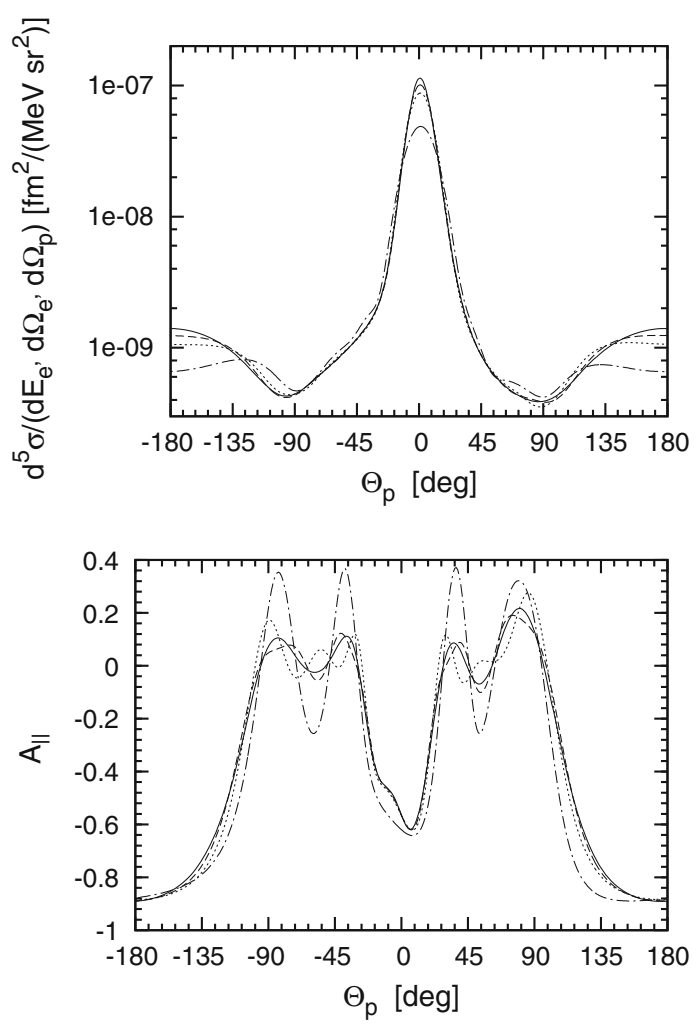

Fig. 12 The same as in Fig. 9 for the $K 6$ electron kinematics from Table 2. Partial wave based results with $j \leq 4$ (dash-dotted line), $j \leq 7$ (dotted line) and $j \leq 9$ (dashed line) are compared with the full 3D prediction (solid line)
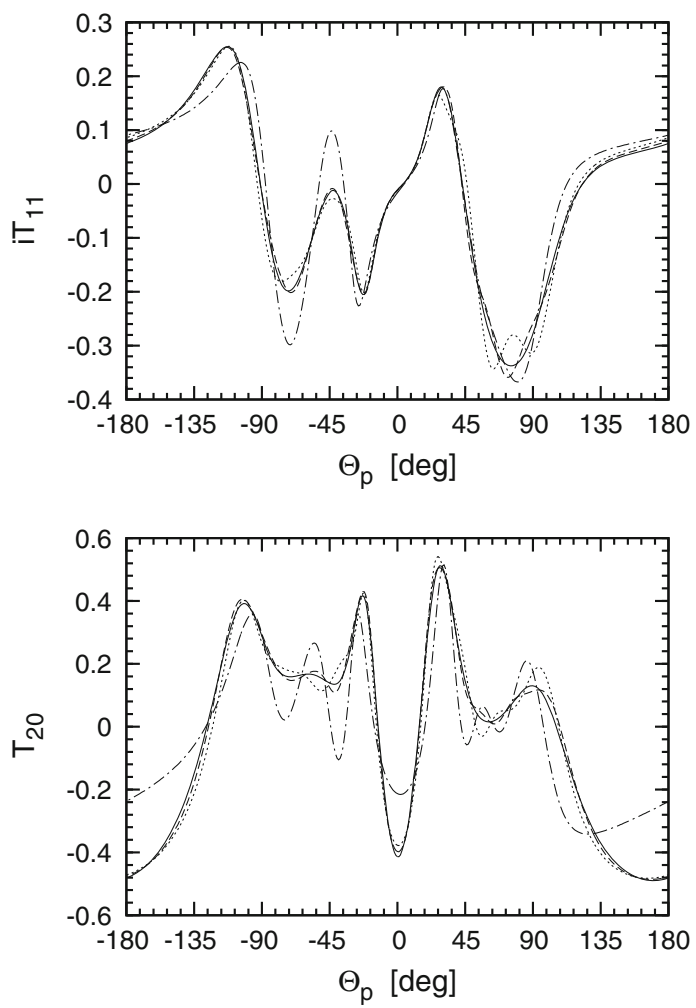

Fig. 13 The same as in Fig. 12 for the deuteron analyzing powers $i T_{11}$ and $T_{20}$ 

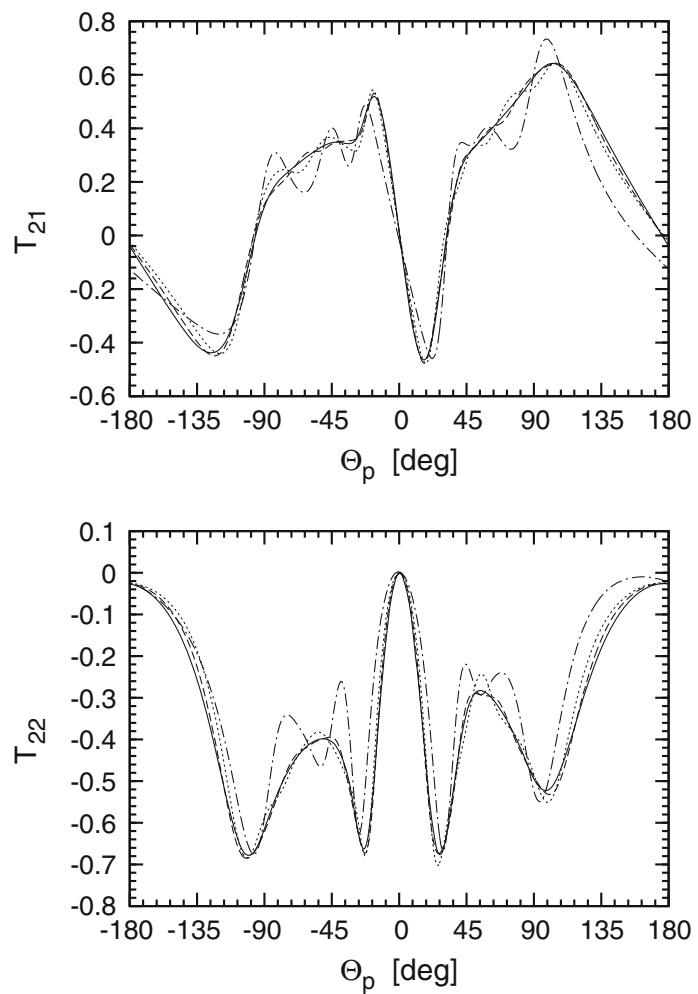

Fig. 14 The same as in Fig. 12 for the deuteron analyzing powers $T_{21}$ and $T_{22}$

where $l\left(l^{\prime}\right)$ is the initial (final) angular momentum of the $2 \mathrm{~N}$ system, $s$ is the $2 \mathrm{~N}$ (conserved) spin and $j$ is the total (conserved) $2 \mathrm{~N}$ angular momentum. These matrix elements can be compared with direct solutions of LSE obtained in the standard partial wave representation. We performed the projection of the 3D t-matrix on partial waves, employing the simple method proposed in Ref. [15] for NN forces. In Figs. 4, 5, 6 and 7 we show examples for the uncoupled and coupled channels, with the $2 \mathrm{~N}$ isospin $t=0$ and $t=1$. We chose $p^{\mathrm{f}} \approx 1.9 \mathrm{fm}^{-1}$, which corresponds to a relatively high $\mathrm{NN}$ center of mass energy, $E_{2 N}=150 \mathrm{MeV}$. For such an energy many partial waves contribute to the NN scattering observables and the question arises if the partial contributions are consistent with the full 3D calculations. From Figs. 4, 5, 6 and 7, where we show t-matrices for a few dominant $2 \mathrm{~N}$ partial waves (and many other cases which are not shown here) we infer that this is really the case. The agreement between results based on the two quite different approaches is very good. In both cases we used 40 Gaussian $p$ points distributed in the $\left(0, \bar{p}=6 \mathrm{fm}^{-1}\right)$ interval. For the intermediate angular integrations in the $3 \mathrm{D}$ calculations we took $50 x$ and $50 \phi$ points.

The final ingredient in our framework is the $2 \mathrm{~N}$ current operator. It consists of the single-nucleon and $2 \mathrm{~N}$ operators. For the purpose of this paper we assume that its single-nucleon part comprises the standard nonrelativistic charge density as well as the convection and spin current operators. In the $2 \mathrm{~N}$ part we take for simplicity only the leading one-pion-exchange current operator in the chiral effective field theory representation. In our 3D treatment of the ${ }^{2} \mathrm{H}\left(e, e^{\prime} p\right) n$ reaction, we calculate the spin and isospin matrix elements of the current operator directly, using simple matrix representations of the spin and isospin operators and the concept of the Kronecker product to deal with the $2 \mathrm{~N}$ spin and isospin spaces, as discussed in Sect. 7. In the traditional calculations, a partial wave decomposition of the current operator is required. It is a rather easy task for the single-nucleon part of the current operator. For the one-pion-exchange current operator it is known analytically (see for instance Ref. [16]). It can also be obtained using the method proposed in Refs. [17,18], where even more complicated two-pion-exchange current operators were considered.

To give examples of our results on deuteron electro disintegration we chose several electron kinematics given in Table 2. They allowed us to study the reaction for three different internal nucleon-nucleon energies (corresponding to the three values of $p^{\mathrm{f}}$ ) and for five values of the three-momentum transfer $Q$. The first parameter is the input for the t-matrix calculations and the second one specifies the properties of the current matrix elements. 

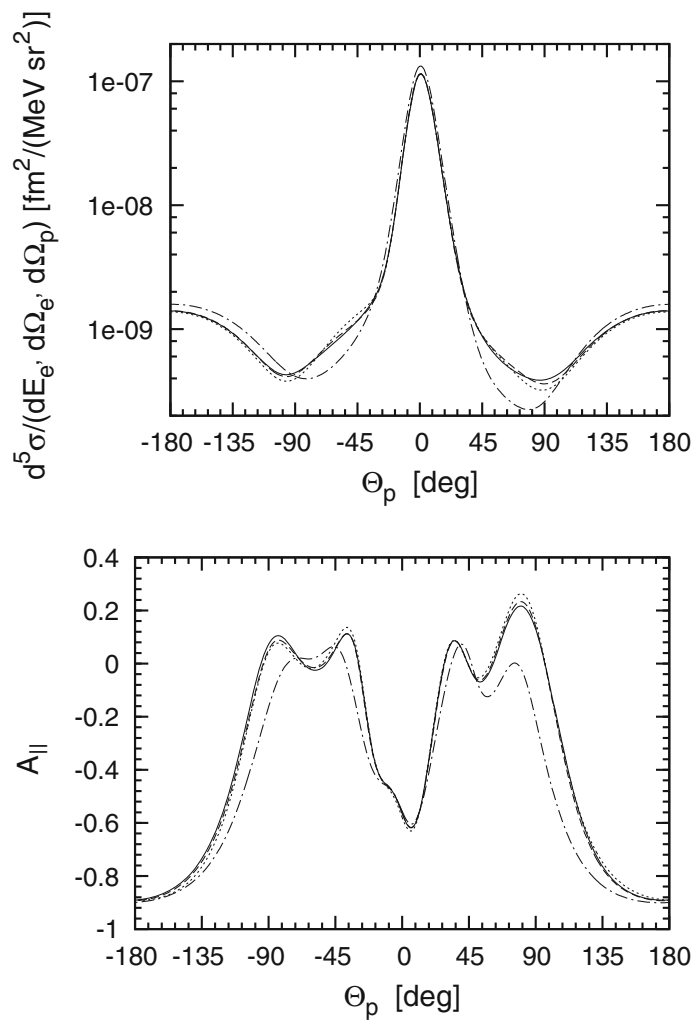

Fig. 15 The unpolarized cross section $d^{5} \sigma /\left(d E_{e^{\prime}} d \Omega_{e^{\prime}} d \Omega_{p}\right)$ and the spin-dependent helicity asymmetry $A_{\|}$as a function of the proton scattering angle $\theta_{p}$ for the $K 6$ electron kinematics from Table 2 . The single-nucleon current contribution to the plane wave part of the nuclear matrix element is now calculated without partial wave decomposition. The remaining parts of the nuclear matrix element are still calculated using partial waves. Results obtained with $j \leq 1$ (dash-dotted line), $j \leq 2$ (dotted line) and $j \leq 3$ (dashed line) are compared with the full 3D prediction (solid line)

In addition to the information given in Table 2 we need to label the exclusive kinematics. For the fixed "electron arm", we deal in fact with a two-body kinematics in the final proton-neutron system. We assume that protons are ejected in the electron plane, where $\theta_{p}$ would be the angle between the three-momentum transfer $\mathbf{Q}$ and the final proton momentum $\mathbf{p}_{p}$. Since we have to distinguish between the $\phi_{p}=0^{\circ}$ and $\phi_{p}=180^{\circ}$ cases, we ascribe the negative sign to $\theta_{p}$ for $\phi_{p}=0^{\circ}$. This is shown in Fig. 8. Note that the six electron kinematics provide a unique solution for any $\theta_{p}$ value and that $\theta_{p}$ changes from 0 to $180^{\circ}$.

We are now ready to show our results for several selected observables. We chose first of all the unpolarized cross section, $d^{5} \sigma /\left(d E_{e^{\prime}} d \Omega_{e^{\prime}} d \Omega_{p}\right)$. We take also into account one example of the spin-dependent helicity asymmetry,

$$
A_{\|} \equiv \frac{\sigma\left(h=+1, \mathbf{J}_{d}\right)-\sigma\left(h=-1, \mathbf{J}_{d}\right)}{\sigma\left(h=+1, \mathbf{J}_{d}\right)+\sigma\left(h=-1, \mathbf{J}_{d}\right)},
$$

where $h$ is the initial electron helicity and the projection of the initial deuteron total angular momentum $\left(\mathbf{J}_{d}\right)$ on $\mathbf{Q}, J_{d z}$, is equal 1. In addition we show our predictions for the deuteron tensor analyzing powers $T_{k q}$. Note that they are calculated in the system, where $\mathbf{Q} \| \hat{z}$.

Our primary goal was to compare results based on the partial wave decomposition for the t-matrix and the nuclear current operator with new predictions resulting from the 3D scheme. We observed a perfect agreement for all the electron kinematics and for all the considered observables, if a sufficient number of partial waves in the first type of calculations is included. The six kinematics can be divided into two groups: $(K 1, K 3$, $K 5)$ and $(K 2, K 4, K 6)$. In each group a similar type of convergence of the observables with respect to the number of partial waves is observed. That is why in Figs. 9, 10,11, 12, 13 and 14 we show predictions for two representative ( $K 3$ and $K 6$ ) kinematics only. In the first case we see a rapid convergence and partial wave based results with $j \leq 4$ are already very close to the full 3D prediction. In the second case all partial waves with $j \leq 9$ are necessary to achieve convergence. 

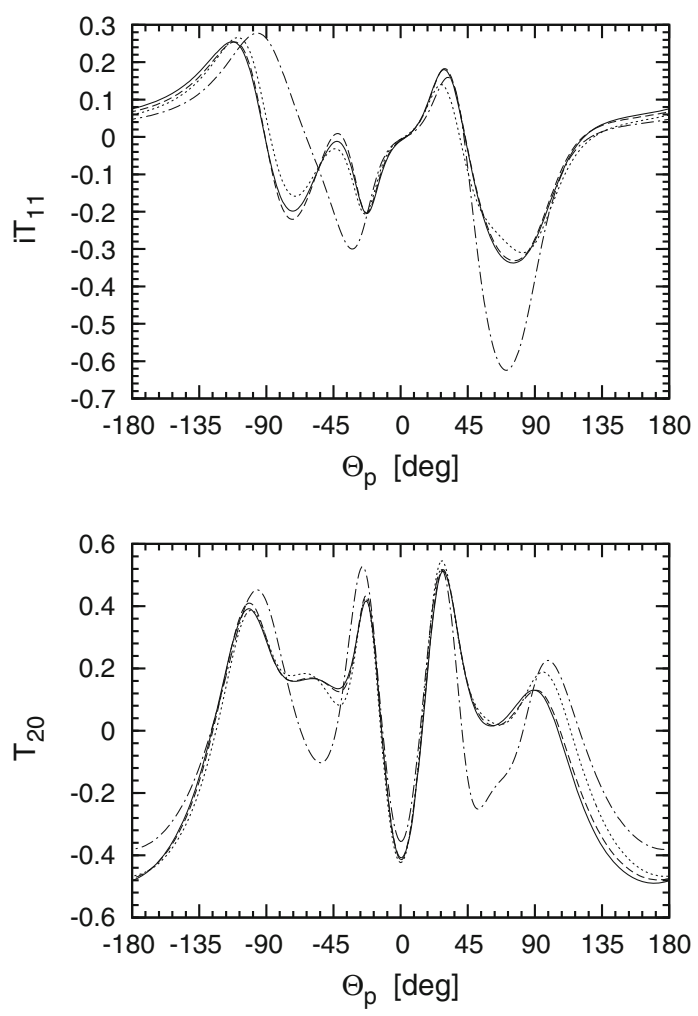

Fig. 16 The same as in Fig. 15 for the deuteron analyzing powers $i T_{11}$ and $T_{20}$
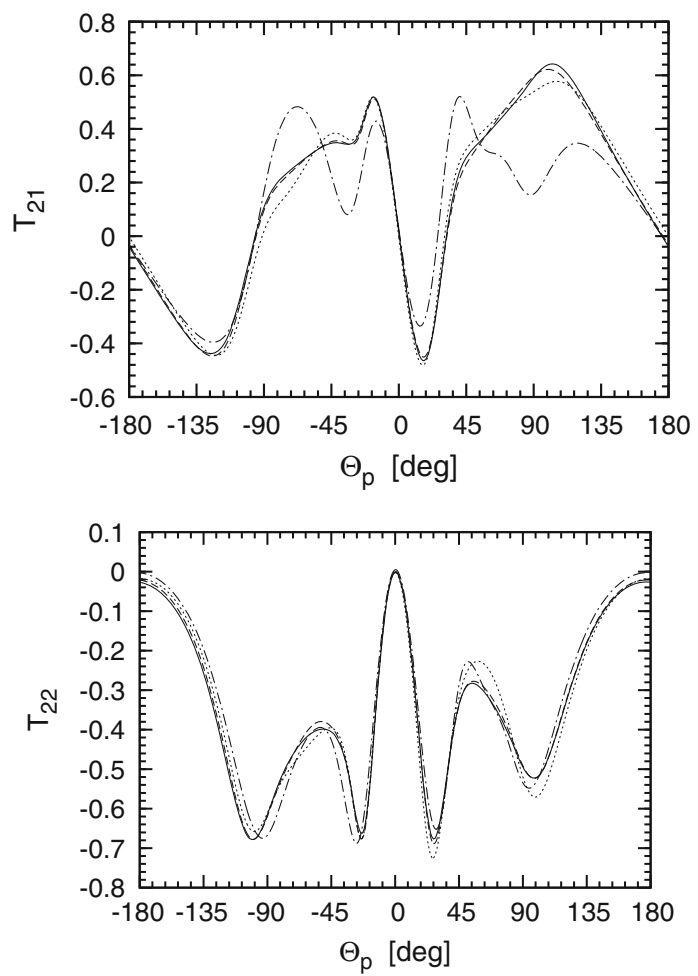

Fig. 17 The same as in Fig. 15 for the deuteron analyzing powers $T_{21}$ and $T_{22}$ 
It is interesting to see that slow convergence for the $K 2, K 4$ and $K 6$ kinematics does not result from the higher $p^{\mathrm{f}}$ values (that is from the t-matrix) but is related to the $Q$ values and thus to the partial wave decomposition of the current operator. It is well known (see for example Ref. [17]) that especially partial wave decomposition of the single nucleon current requires many partial waves. However, even if the initial bound state is given in the partial wave representation, the single nucleon current can be applied directly in the case of the plane wave amplitudes. This holds not only for the two- but also for the three-nucleon system [19]. In order to demonstrate this behavior, we showed in Figs. 15, 16 and 17 observables for the $K 6$ kinematics. In this case the single-nucleon current contribution to the plane wave amplitude is calculated without partial wave decomposition. We see clearly that the convergence is significantly improved, even if the $2 \mathrm{~N}$ current contribution to the plane wave part of the nuclear matrix element and the whole rescattering part of the nuclear matrix element is calculated with the partial wave decomposition.

\section{Conclusions and Outlook}

The presented method to treat several electroweak processes involving $1 \mathrm{~N}$ and $2 \mathrm{~N}$ current operators in three dimensions can successfully replace standard partial wave treatment. We showed, for the case of electron induced deuteron disintegration, that results obtained using the new approach agree very well with those obtained using PWD. For all observables considered in this paper, the traditional results converge to the 3D predictions when the number of partial waves is sufficiently high (Figs. 9, 10, 11, 12, 13, 14).

Our formalism employs the two nucleon bound state, the $2 \mathrm{~N} t$ matrix and the current operators in the joined isospin-spin space of the $2 \mathrm{~N}$ system using $3 \mathrm{D}$ formalism in the $2 \mathrm{~N}$ momentum space. Each element of this framework has been separately tested and compared with the standard PWD approach. Our method seems to be more flexible and can deal with the rich structures of the $2 \mathrm{~N}$ force and the current operator, especially derived within the higher orders of the chiral effective field theory $[11,13,20]$. We plan to use our framework for other processes such as muon capture or neutrino induced deuteron disintegration. In [2, references therein] muon capture on ${ }^{2} \mathrm{H}$ and ${ }^{3} \mathrm{He}$ was considered with the use of the PWD approach. It would be interesting to compare those results with 3D calculations. A similar convergence to 3D results as in Figs. 9, 10, 11, 12, 13 and 14 is expected.

Acknowledgments We acknowledge support by the Foundation for Polish Science - MPD program, co-financed by the European Union within the Regional Development Fund. This work was supported by the Polish National Science Center under Grant No. DEC-2011/01/B/ST2/00578 and partially by the EU HadronPhysics3 project "Exciting Physics Of Strong Interactions". One of the authors (JG) would like to thank K. Sagara for the hospitality extended to him during his stay at the Kyushu University and E. Epelbaum for the hospitality extended to him during his stay at the Ruhr-Universität, Bochum. KT would like to thank Ulf-G. Meißner for the hospitality extended to him during his stay at the Institut für Kernphysik in the Forschungszentrum Jülich.

Open Access This article is distributed under the terms of the Creative Commons Attribution License which permits any use, distribution, and reproduction in any medium, provided the original author(s) and the source are credited.

\section{References}

1. Wolfram Research Inc.: Mathematica version 8.0. Wolfram Research Inc. Champaign, Illinois (2010)

2. Marcucci, L.E., Piarulli, M., Viviani, M., Girlanda, L., Kievsky, A., et al.: Muon capture on deuteron and ${ }^{3}$ He. Phys. Rev. C 83, 014002 (2011)

3. Shen, G., Marcucci, L.E., Carlson, J., Gandolfi, S., Schiavilla, R.: Inclusive neutrino scattering off deuteron from threshold to $\mathrm{GeV}$ energies. arXiv:1205.4337v1 [nucl-th] (2012)

4. Lalakulich, O., Gallmeister, K., Mosel, U.: Neutrino and antineutrino induced reactions with nuclei between 1 and $30 \mathrm{GeV}$. arXiv:1205.1061v2 [nucl-th] (2012)

5. Ericson, T., Weise, W.: Pions and nuclei. Int. Ser. Monographs Phys. 74, 49 (1998)

6. Fachruddin, I., Elster, Ch., Glöckle, W.: New forms of deuteron equations and wave function representations. Phys. Rev. C 63, 054003 (2001)

7. Golak, J., Glöckle, W., Skibiński, R., Witała, H., Rozpędzik, D., Topolnicki, K., Fachruddin, I., Elster, Ch., Nogga, A.: The two-nucleon system in three dimensions. Phys. Rev. C 81, 034006 (2010)

8. Riska, D.O.: Isovector electromagnetic exchange currents and the nucleon-nucleon interaction. Physica Scripta 31, 471 (1985)

9. Schiavilla, R., Pandharipande, V.R., Riska, D.O.: Magnetic form factors of the trinucleons. Phys. Rev. C40, 2294-2309 (1989)

10. Schiavilla, R., Pandharipande, V.R., Riska, D.O.: Charge form factors of the three- and four-body nuclei. Phys. Rev. C 41, 309317 (1990)

11. Kölling, S., Epepbaum, E., Krebs, H., Meißner, U.G.: Two-pion exchange electromagnetic current in chiral effective field theory using the method of unitary transformation. Phys. Rev. C 80, 045502 (2009) 
12. Wolfenstein, L.: Possible triple-scattering experiments. Phys. Rev. 96, 1654 (1954)

13. Epelbaum, E.: Few-nucleon forces and systems in chiral effective field theory. Prog. Part. Nucl. Phys. 57, 654-741 (2006)

14. Press, W., Flannery, B., Teukolsky, S., Vetterling, W.: Numerical Recipes. Cambridge University Press, Cambridge (1989)

15. Golak, J., Rozpȩdzik, D., Skibiński, R., Topolnicki, K., Witała, H., Glöckle, W., Nogga, A., Epelbaum, E., Kamada, H., Elster, Ch., Fachruddin, I.: A new way to perform partial wave decompositions of few-nucleon forces. Eur. Phys. J. A 43, 241 (2010)

16. Kotlyar, V.V., Kamada, H., Glöckle, W., Golak, J.: Partial wave decomposition for meson exchange currents in few-nucleon systems. Few-Body Syst. 28, 35 (2000)

17. Rozpędzik, D.: Unpublished Phd thesis, Jagiellonian University, Cracow (2010)

18. Rozpȩdzik, D., Golak, J., Kölling, S., Epelbaum, E., Skibiński, R., Witała, H., Krebs, H.: Signatures of the chiral two-pion exchange electromagnetic currents in the ${ }^{2} \mathrm{~h}$ and ${ }^{3}$ he photodisintegration reactions. Phys. Rev. C 83, 064004 (2011)

19. Golak, J., Skibiński, R., Witała, H., Glöckle, W., Nogga, A., Kamada, H.: Electron and photon scattering on three-nucleon bound states. Phys. Rept. 415, 89-205 (2005)

20. Kölling, S., Epepbaum, E., Krebs, H., Meißner, U.G.: Two-nucleon electromagnetic current in chiral effective field theory: one pion exchange and short-range contributions. Phys. Rev. C 84, 054008 (2011) 